\title{
Routh Reduction by Stages
}

Bavo LANGEROCK ${ }^{\dagger \ddagger \S}$, Tom MESTDAG ${ }^{\dagger}$ and Joris VANKERSCHAVER ${ }^{\dagger \star}$

$\dagger$ Department of Mathematics, Ghent University, Krijgslaan 281, S22, B9000 Ghent, Belgium E-mail: bavo.langerock@ugent.be,tom.mestdag@ugent.be,joris.vankerschaver@ugent.be

¥Belgian Institute for Space Aeronomy, Ringlaan 3, B1180 Brussels, Belgium

$\S$ Department of Mathematics, K.U. Leuven, Celestijnenlaan 200 B, B3001 Leuven, Belgium

* Department of Mathematics, University of California at San Diego, 9500 Gilman Drive, San Diego CA 92093-0112, USA

Received June 16, 2011, in final form November 22, 2011; Published online November 29, 2011 http://dx.doi.org/10.3842/SIGMA.2011.109

Abstract. This paper deals with the Lagrangian analogue of symplectic or point reduction by stages. We develop Routh reduction as a reduction technique that preserves the Lagrangian nature of the dynamics. To do so we heavily rely on the relation between Routh reduction and cotangent symplectic reduction. The main results in this paper are: (i) we develop a class of so called magnetic Lagrangian systems and this class has the property that it is closed under Routh reduction; (ii) we construct a transformation relating the magnetic Lagrangian system obtained after two subsequent Routh reductions and the magnetic Lagrangian system obtained after Routh reduction w.r.t. to the full symmetry group.

Key words: symplectic reduction; Routh reduction; Lagrangian reduction; reduction by stages

2010 Mathematics Subject Classification: 37J05; 37J15; 52D20

\section{Contents}

1 Introduction $\quad 2$

2 Preliminaries on Routh reduction 2

3 Magnetic Lagrangian systems $\quad 6$

4 Magnetic Lagrangian systems with symmetry 9

4.1 Symplectic reduction: a brief introduction . . . . . . . . . . . . . . . . 9

4.2 Invariant magnetic Lagrangian systems and momentum maps . . . . . . . . . . . . . . 9

5 Routh reduction for magnetic Lagrangian systems $\quad 11$

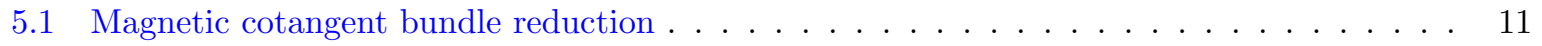

5.2 Routh reduction for magnetic Lagrangian systems . . . . . . . . . . . . . . . . . . . 14

5.3 Reduction of magnetic Lagrangian systems on Lie groups . . . . . . . . . . . . . . 18

6 Routh reduction by stages $\quad 19$

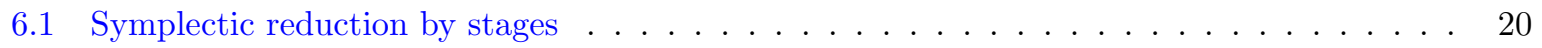

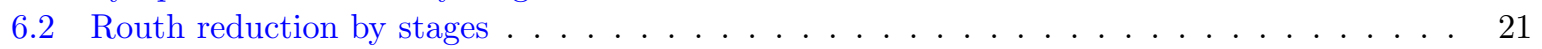

7 Examples $\quad 25$

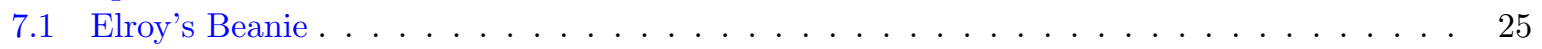

7.2 Rigid bodies on the Heisenberg group $\ldots \ldots \ldots \ldots \ldots \ldots \ldots$

$\begin{array}{ll}\text { References } & 30\end{array}$ 


\section{Introduction}

It is not a surprise that the bigger part of the recent literature on the geometric description of mechanical systems deals in some way or another with symmetry. The benefits of exploiting symmetry are indeed self-evident: The equations of motion of a mechanical system which exhibits a symmetry can be reduced to a new set of equations with fewer unknowns, possibly easier to solve. In particular, the Hamiltonian description of such systems has attracted most of the attention in the literature, and the important role played by Poisson manifolds and symplectic structures has been strongly emphasized (see e.g. [12] and references therein). Less well-known is the process of symmetry reduction for Lagrangian systems. Much like on the Hamiltonian side, there are in fact two different paths that lead to two different Lagrangian reduction theories. Roughly speaking, the invariance of the Lagrangian leads via Noether's theorem to a set of conserved quantities (the momenta). Whether or not one takes these conserved quantities into account in the reduction process leads to either the Routh or the Lagrange-Poincaré reduction method (see e.g. [4, 5, 6, 10, 14, 15]).

In this paper we deal with Routh's reduction procedure. In a way, one restricts the attention to only those solutions of the system with a prescribed value of momentum. The price one has to pay is that the new symmetry group (after restriction) is in general only a subgroup of the symmetry group of the original Lagrangian system. In [10] it was pointed out that Routh reduction can be interpreted as a special case of symplectic reduction, which moreover preserves the Lagrangian nature of the system.

In the current paper we will investigate those aspects of the theory that are related to reduction in multiple stages. In case the symmetry group of the system has a normal subgroup, one can indeed first perform a Routh reduction by means of this subgroup. It is then natural to ask whether the reduced system is invariant under some residual, as yet to be determined group, and whether a subsequent second Routh reduction leads to results which are equivalent to direct reduction by the full group. The answer to the same question, but for the above mentioned Lagrange-Poincaré reduction theory, is by now well-known [4]. It can be understood in the following sense: After each reduction step one remains in the category of so-called 'Lagrangian systems on Lie algebroids' and symmetry reduction can be phrased in terms of Lie algebroid morphisms (see e.g. [5] and the references therein). Equivalently, one may use a connection to decompose the Lie algebroid structure at each stage. The corresponding category is then the so-called category of 'Lagrange-Poincaré bundles' from [4], while the corresponding 'Lagrange-Poincaré morphisms' do the reduction.

It is natural to ask whether or not such a category of systems exists also for Routh reduction (it is mentioned as an open problem in [12]). The candidate we propose (in Section 3) is the class of what we call 'magnetic Lagrangian systems'. We show in Sections 4 and 5 that this class has the property that it is closed under Routh reduction, i.e. that after each step of a reduction in stages the reduced system remains in the class of magnetic Lagrangian systems. As in [10], we heavily rely on a generalized version of cotangent symplectic reduction. In Section 6 we apply the framework of magnetic Lagrangian systems to reduction in several stages, where the first stage consists of Routh reduction by a normal subgroup of the overall symmetry group. We end the paper with some illustrative examples in the final section.

\section{Preliminaries on Routh reduction}

Definition 1. A Lagrangian system is a pair $(Q, L)$ with $Q$ a manifold and $L$ a function on $T Q$. The manifold $Q$ is the configuration space and $L$ is called the Lagrangian. The dimension of $Q$ is called the number of degrees of freedom of the Lagrangian system. A Lagrangian system is 
of mechanical type if for arbitrary $v_{q} \in T_{q} Q$, the Lagrangian can be written as

$$
L\left(v_{q}\right)=\frac{1}{2}\left\langle\left\langle v_{q}, v_{q}\right\rangle\right\rangle_{Q}-V(q),
$$

with $\langle\langle\cdot, \cdot\rangle\rangle_{Q}$ a Riemannian metric on $Q$ and $V$ a function on $Q$, called the potential energy of the Lagrangian. The function $\frac{1}{2}\left\langle\left\langle v_{q}, v_{q}\right\rangle_{Q}\right.$ is called the kinetic energy.

We are interested in the Euler-Lagrange equations associated to a Lagrangian system. These equations are necessary conditions for a curve $q(t): I \subset \mathbb{R} \rightarrow Q$ to extremize the action integral $\int_{I} L(q(t), \dot{q}(t)) d t$. In a local coordinate chart $\left(q^{i}\right)$ on $Q$ the Lagrangian $L$ is a function of $\left(q^{i}, v^{i}\right)$ and the Euler-Lagrange equations are

$$
\frac{d}{d t}\left(\frac{\partial L}{\partial v^{i}}\right)-\frac{\partial L}{\partial q^{i}}=0, \quad \dot{q}^{i}=v^{i}, \quad i=1, \ldots, n=\operatorname{dim} Q .
$$

Routh reduction is a reduction technique for Lagrangian systems that are invariant under the action of a symmetry group. In classical textbooks, it is a step-by-step procedure that describes the construction of a new Lagrangian function with fewer degrees of freedom.

Before we can formulate Routh reduction, we fix notations for the action of a Lie group on an arbitrary manifold $M$ and related concepts.

Actions of Lie groups and principal bundles. When a Lie group $G$ is given, $\mathfrak{g}$ denotes the Lie algebra of $G$ and exp the exponential map from $\mathfrak{g}$ to $G$. The adjoint action of $G$ on $\mathfrak{g}$ is denoted by Ad and the coadjoint action of $G$ on $\mathfrak{g}^{*}$ by $\mathrm{Ad}^{*}$.

\section{Definition 2.}

1. A right action of a group $G$ on a manifold $M$ is denoted by $\Psi^{M}: G \times M \rightarrow M ;(g, m) \mapsto$ $\Psi_{g}^{M}(m)=m g$. Throughout this paper we only consider free and proper actions of Lie groups on manifolds.

2. The infinitesimal action is given by $\psi_{m}^{M}: \mathfrak{g} \rightarrow T_{m} M ; \xi \mapsto d /\left.d \epsilon\right|_{0}(m \exp \epsilon \xi)$.

3. For any element $\xi \in \mathfrak{g}$ one can consider the fundamental vector field $\xi_{M}$, defined pointwise as $\xi_{M}(m)=\psi_{m}^{M}(\xi)$.

4. The map defined pointwise as the dual to $\psi_{m}^{M}$ is denoted by $\left(\psi^{M}\right)^{*}: T^{*} M \rightarrow \mathfrak{g}^{*}$.

5. The lifted action $\Psi^{T M}$ on $T M$ of $\Psi^{M}$ is given by $\Psi^{T M}: G \times T M \rightarrow T M ;\left(g, v_{m}\right) \mapsto$ $T \Psi_{g}^{M}\left(v_{m}\right)$.

6. The lifted action $\Psi^{T^{*} M}$ on $T^{*} M$ is given by $\Psi^{T^{*} M}: G \times T^{*} M \rightarrow T^{*} M ;\left(g, \alpha_{m}\right) \mapsto$ $T^{*} \Psi_{g^{-1}}^{M}\left(\alpha_{m}\right)$.

With these notations, $\xi_{T M}$ denotes a fundamental vector field on $T M$ determined by the lifted action. It follows that $\xi_{T M}$ is the complete lift of $\xi_{M}$.

Every right action $\Psi^{M}$ gives rise to a left action $\Phi^{M}: \Phi_{g}^{M}(m)=\Psi_{g^{-1}}^{M}(m)$. We only consider right actions. This is not a true restriction since one may reformulate the main results for left actions if needed.

The orbit space $M / G$ of a free and proper action is a manifold and $\pi: M \rightarrow M / G$ carries the structure of a principal $G$-bundle. Throughout the paper $[m]_{G}$ denotes a point in the orbit space $M / G$, i.e. it is the orbit through $m \in M$. Every tangent vector in the kernel of $T \pi$ is of the form $\xi_{M}(m)$ for some $\xi \in \mathfrak{g}$. These vectors are called vertical and form a distribution which we call the vertical distribution $V \pi=\operatorname{ker} T \pi$.

Definition 3. A principal connection on a principal $G$-bundle is a $\mathfrak{g}$-valued 1-form $\mathcal{A}$ satisfying two conditions: 
1) it is equivariant, i.e. $\Psi_{g}^{*} \mathcal{A}=\operatorname{Ad}_{g^{-1}} \cdot \mathcal{A}$ for any $g \in G$, and

2) for $\xi \in \mathfrak{g}$ arbitrary, $\mathcal{A}\left(\xi_{M}\right)=\xi$.

The kernel of $\mathcal{A}$ determines a right invariant distribution on $M$ which is a complement of the vertical distribution. It is therefore called the horizontal distribution of $\mathcal{A}$ and is typically denoted by $H^{\mathcal{A}} \subset T M$. On the other hand, any right invariant distribution $H$ satisfying $H \oplus V \pi=T Q$ determines a principal connection, see [8].

We denote by $\langle f, \mathcal{A}\rangle$, where $f$ is a $\mathfrak{g}^{*}$-valued function on $M$, the 1 -form on $M$ pointwise defined by

$$
v_{m} \mapsto\left\langle f(m), \mathcal{A}(m)\left(v_{m}\right)\right\rangle \in \mathbb{R} .
$$

The cotangent vector $\langle f, \mathcal{A}\rangle(m) \in T_{m}^{*} M$ is often denoted by $\langle f(m), \mathcal{A}(m)\rangle$. In particular, if $f=\mu \in \mathfrak{g}^{*}$ is constant, then $\langle\mu, \mathcal{A}\rangle$ is a 1 -form on $M$.

\section{Routh reduction.}

Definition 4. Let $(Q, L)$ denote a Lagrangian system and assume that the configuration space is equipped with an action $\Psi^{Q}$. The Lagrangian system $(Q, L)$ is $G$-invariant if $L$ is invariant under the lifted action $\Psi^{T Q}$, i.e. $L\left(\Psi_{g}^{T Q}\left(v_{q}\right)\right)=L\left(v_{q}\right)$ for arbitrary $v_{q} \in T Q$ and $g \in G$.

If a mechanical Lagrangian system is $G$-invariant then the kinetic energy metric $\left\langle\langle\cdot, \cdot\rangle_{Q}\right.$ and the potential energy $V$ are both invariant under the pull-back of $\Psi_{g}^{Q}$, for arbitrary $g$.

\section{Definition 5.}

1. For a Lagrangian system $(Q, L)$, the Legendre transform $\mathbb{F} L: T Q \rightarrow T^{*} Q$ is the fibre derivative of $L$, i.e. for arbitrary $v_{q}, w_{q} \in T Q$

$$
\left\langle\mathbb{F} L\left(v_{q}\right), w_{q}\right\rangle=\left.\frac{d}{d u}\right|_{u=0} L\left(v_{q}+u w_{q}\right) .
$$

The Lagrangian is hyperregular if $\mathbb{F} L$ is a diffeomorphism.

2. For a $G$-invariant Lagrangian system $(Q, L)$, the momentum map $J_{L}: T Q \rightarrow \mathfrak{g}^{*}$ is the map $\left(\psi^{Q}\right)^{*} \circ \mathbb{F} L$, i.e. for arbitrary $v_{q} \in T Q$ and $\xi \in \mathfrak{g}$

$$
\left\langle J_{L}\left(v_{q}\right), \xi\right\rangle=\left\langle\mathbb{F} L\left(v_{q}\right), \xi_{Q}(q)\right\rangle .
$$

3. Given a $G$-invariant mechanical Lagrangian system $(Q, L)$, and a point $q$ in $Q$. The inertia tensor $\mathbb{I}_{q}$ is a metric on $\mathfrak{g}$ defined by $\mathbb{I}_{q}(\xi, \eta)=\left\langle\left\langle\xi_{Q}(q), \eta_{Q}(q)\right\rangle_{Q}\right.$.

The momentum map associated to a $G$-invariant Lagrangian system satisfies the following two important properties:

1. It is conserved along the solutions of the Euler-Lagrange equations, i.e. if $q(t)$ is a solution to the Euler-Lagrange equations, then $\frac{d}{d t}\left(J_{L}(\dot{q}(t))\right)=0$.

2. It is equivariant w.r.t. the action $\Psi^{T Q}$ and the coadjoint action $\operatorname{Ad}^{*}$ on $\mathfrak{g}^{*}$, i.e.

$$
J_{L}\left(\Psi_{g}^{T Q}\left(v_{q}\right)\right)=\operatorname{Ad}_{g}^{*}\left(J_{L}\left(v_{q}\right)\right)
$$

for arbitrary $g \in G$ and $v_{q} \in T Q$.

Definition 6. Given a $G$-invariant Lagrangian system $(Q, L)$ and an arbitrary vector $v_{q} \in T Q$.

1. The map $\left.J_{L}\right|_{v_{q}}: \mathfrak{g} \rightarrow \mathfrak{g}^{*}$ is the $\operatorname{map} \xi \mapsto J_{L}\left(v_{q}+\xi_{Q}(q)\right)$.

2. The Lagrangian system $(Q, L)$ is $G$-regular if $\left.J_{L}\right|_{v_{q}}$ is a diffeomorphism for every $v_{q} \in T Q$. 
Every $G$-invariant mechanical Lagrangian system is $G$-regular. To show this, remark that for a mechanical Lagrangian system

$$
\left\langle\left. J_{L}\right|_{v_{q}}(\xi), \eta\right\rangle=\left\langle\left\langle v_{q}, \eta_{Q}(q)\right\rangle\right\rangle_{Q}+\left\langle\left\langle\xi_{Q}(q), \eta_{Q}(q)\right\rangle_{Q} .\right.
$$

Hence $\left.J_{L}\right|_{v_{q}}(\xi)=\left.J_{L}\right|_{v_{q}}(0)+\mathbb{I}_{q}(\xi)$ is an affine map, whose linear part is determined by the inertia metric on $\mathfrak{g}$.

Proposition 1. Consider a $G$-invariant and $G$-regular Lagrangian system $(Q, L)$ and fix a regular value $\mu \in \mathfrak{g}^{*}$ of the momentum map $J_{L}$. Let $G_{\mu}$ denote the isotropy subgroup of $\mu$ w.r.t. the coadjoint action of $G$ on $\mathfrak{g}^{*}$, i.e. $g \in G_{\mu}$ iff $\operatorname{Ad}_{g}^{*} \mu=\mu$.

1. The submanifold $i_{\mu}: J_{L}^{-1}(\mu) \rightarrow T Q$ is $G_{\mu}$-invariant and the restricted action of $G_{\mu}$ on $J_{L}^{-1}(\mu)$ is free and proper.

2. The quotient manifold $J_{L}^{-1}(\mu) / G_{\mu}$ is diffeomorphic to the fibred product $T(Q / G) \times_{Q / G}$ $Q / G_{\mu}$ over $Q / G$.

The first statement is a direct consequence of the equivariance of $J_{L}$. We postpone a proof of the second statement (Proposition 7, page 15).

We are now ready to describe a preliminary version of Routh reduction where the symmetry group $G$ is Abelian and the bundle $Q \rightarrow Q / G$ is trivial, so that we can choose a connection $\mathcal{A}$ with vanishing curvature. Since $G$ is Abelian, we have that the isotropy subgroup $G_{\nu}$ for every $\nu \in \mathfrak{g}^{*}$ is the entire group $G$, and in particular $T(Q / G) \times_{Q / G} Q / G_{\nu}$ is nothing but $T(Q / G)$.

Theorem 1 (Routh reduction - Abelian version). Let $G$ be Abelian and let $\mathcal{A}$ be a connection on $Q$ with vanishing curvature. The Routh reduction procedure of a $G$-invariant and $G$-regular Lagrangian system $(Q, L)$ consists of the following steps.

1. Fix a regular value $\mu$ of the momentum map $J_{L}$ and consider the submanifold $J_{L}^{-1}(\mu)$.

2. Compute the restriction of the $G$-invariant function $L-\langle\mu, \mathcal{A}\rangle$ to the level set $J_{L}^{-1}(\mu)$. Let $\tilde{L}$ denote its projection to the quotient manifold $T(Q / G) \cong J_{L}^{-1}(\mu) / G$.

Every solution of the Euler-Lagrange equations of the Lagrangian system $(Q / G, \tilde{L})$ is the projection of a solution of the Euler-Lagrange equations of the original system $(Q, L)$ with momentum $\mu$. Conversely, every solution of the Euler-Lagrange equations of the Lagrangian system $(Q, L)$ with momentum $\mu$ projects to a solution of the Euler-Lagrange equations of the system $(Q / G, \tilde{L})$.

One may also find a description of global Abelian Routh reduction in [3].

Example: the spring pendulum. The system consists of a point particle with mass $m$ moving in a horizontal plane, and attached to the origin by means of a spring with spring constant $k$. We choose polar coordinates $(r, \theta)$ for this system, so that the mechanical Lagrangian is given by $L=\frac{1}{2} m\left(\dot{r}^{2}+r^{2} \dot{\theta}^{2}\right)-\frac{1}{2} k r^{2}$. This system is clearly invariant under translations in the $\theta$-direction, given by $\Psi_{a}(r, \theta)=(r, \theta+a)$. The momentum map for this action is $J_{L}=m r^{2} \dot{\theta}$ and the Euler-Lagrange equations are

$$
\begin{aligned}
& m \ddot{r}-m r \dot{\theta}^{2}+k r=0, \\
& \frac{d}{d t}\left(m r^{2} \dot{\theta}\right)=0 .
\end{aligned}
$$

Let us fix a regular value $0 \neq \mu=m r^{2} \dot{\theta}$ for the momentum map and let $\mathcal{A}=d \theta$ be the standard connection with vanishing curvature. The Routhian is obtained from

$$
\tilde{L}(r, \dot{r})=\left.(L-\mu \dot{\theta})\right|_{m r^{2} \dot{\theta}=\mu}=\frac{1}{2} m \dot{r}^{2}-\frac{1}{2} k r^{2}-\frac{1}{2} \frac{\mu^{2}}{m r^{2}} .
$$


The Euler-Lagrange equation for $\tilde{L}$ is $m \ddot{r}=-k r+\frac{\mu^{2}}{m r^{3}}$ and solutions of this equations are in correspondence to solutions of the Euler-Lagrange equations for $L$ with momentum $\mu$.

Routh reduction can be extended to more general contexts, including non-Abelian group actions [14], Lagrangians of non-mechanical type [2, 6], Lagrangians invariant up to a total time derivative [10] and Lagrangians that are not $G$-regular [11]. Additional complications arise in these cases: for instance, if the bundle $Q \rightarrow Q / G$ is not trivial, the reduced system is typically subjected to an additional force term associated to the curvature of the chosen connection. On the other hand, if the symmetry group is not Abelian, the quotient space $J_{L}^{-1}(\mu) / G_{\mu}$ no longer has the structure of a tangent bundle; instead it is diffeomorphic to $T(Q / G) \times_{Q / G} Q / G_{\mu}$. The interpretation of the reduced system as a Lagrangian system then requires additional definitions, which are postponed to the following section.

Our main interest in this paper is Routh reduction by stages, where the assumption is that the Lagrangian system obtained after applying a first Routh reduction carries additional symmetry, so that we can reapply Routh reduction. The system obtained after one Routh reduction is formulated on a fibred product of the type $T(Q / G) \times{ }_{Q / G} Q / G_{\mu}$. This is the fibred product of a bundle $Q / G_{\mu} \rightarrow Q / G$ with the tangent bundle to the base space. This observation is our main motivation to extend Routh reduction to Lagrangian systems defined on such fibred products: in order to develop Routh reduction by stages, we have to be able to reduce Lagrangian systems obtained after a first reduction.

Routh reduction is closely related to symplectic reduction on the associated cotangent bundles (see $[10,14]$ ). In the next section we will introduce the concept of a "magnetic Lagrangian system" and we will emphasize its symplectic formulation. The concept of magnetic Lagrangian systems is the analogue of Hamiltonian systems one encounters in magnetic cotangent bundle reduction [12].

\section{Magnetic Lagrangian systems}

A magnetic Lagrangian system is a Lagrangian system with configuration space the total space of a bundle $\epsilon: P \rightarrow Q$ and where the Lagrangian is independent of the velocities tangent to the fibres of $\epsilon$. Additionally the system is subjected to a force term that is of magnetic type. It might help to keep in mind that in the case of a Routh reduced Lagrangian system, $P$ corresponds to $Q / G_{\mu}$ and the fibration $\epsilon$ is given by the projection $Q / G_{\mu} \rightarrow Q / G$.

Definition 7. A magnetic Lagrangian system is a triple $(\epsilon: P \rightarrow Q, L, \mathcal{B})$ where $\epsilon: P \rightarrow Q$ is a fibre bundle, $L$ is a smooth function on the fibred product $T Q \times{ }_{Q} P$ and $\mathcal{B}$ is a closed 2 -form on $P$. We say that $P$ is the configuration manifold of the system and that $L$ is the Lagrangian.

A coordinate chart $\left(q^{i}, p^{a}\right), i=1, \ldots, n=\operatorname{dim} Q, a=1, \ldots, k=\operatorname{dim} P-\operatorname{dim} Q$, adapted to the fibration $\epsilon: P \rightarrow Q$ determines a coordinate chart $\left(q^{i}, v^{i}, p^{a}\right)$ on $T Q \times{ }_{Q} P$, and the Lagrangian $L$ is then a function depending on $\left(q^{i}, v^{i}, p^{a}\right)$. By definition, $L$ is independent of the velocities in the fibre coordinates $p^{a}$ and therefore it determines a singular Lagrangian when interpreted as a function on $T P$. Locally, the Euler-Lagrange equations for this singular Lagrangian are

$$
\begin{aligned}
& \frac{d}{d t}\left(\frac{\partial L}{\partial v^{i}}\right)-\frac{\partial L}{\partial q^{i}}=\mathcal{B}_{i j} \dot{q}^{j}+\mathcal{B}_{i a} \dot{p}^{a}, \quad i=1, \ldots, n, \\
& -\frac{\partial L}{\partial p^{a}}=-\mathcal{B}_{i a} \dot{q}^{i}+\mathcal{B}_{a b} \dot{p}^{b}, \quad a=1, \ldots, k .
\end{aligned}
$$

Here we used the following coordinate expression of the 2 -form $\mathcal{B}$ is $\frac{1}{2} \mathcal{B}_{i j} d q^{i} \wedge d q^{j}+\mathcal{B}_{i a} d q^{i} \wedge$ $d p^{a}+\frac{1}{2} \mathcal{B}_{a b} d p^{a} \wedge d p^{b}$. These Euler-Lagrange equations have a geometric interpretation (see Proposition 2). First we introduce additional notations. 
Definition 8. Assume a magnetic Lagrangian system $(\epsilon: P \rightarrow Q, L, \mathcal{B})$ is given.

1. $T_{P} Q$ denotes the fibred product $T Q \times{ }_{Q} P$ and $\left(v_{q}, p\right)$, where $v_{q} \in T Q$ and $p \in P$ such that $\epsilon(p)=q$, is a point in $T_{P} Q$. Similarly, $T_{P}^{*} Q$ denotes the fibred product $T^{*} Q \times{ }_{Q} P$ and $\left(\alpha_{q}, p\right)$, with $\epsilon(p)=q$, is an arbitrary element in $T_{P}^{*} Q$.

2. $V \epsilon$ denotes the distribution on $P$ of tangent vectors vertical to $\epsilon$.

3. $\hat{\epsilon}: T P \rightarrow T_{P} Q$ is the projection fibred over $P$ that maps $v_{p} \in T P$ onto $\left(T \epsilon\left(v_{p}\right), p\right) \in T_{P} Q$.

4. $\rho_{1}: T_{P} Q \rightarrow T Q$ is the projection that maps $\left(v_{q}, p\right) \in T_{P} Q$ onto $v_{q} \in T Q$.

5. $\rho_{2}: T_{P} Q \rightarrow P$ is the projection that maps $\left(v_{q}, p\right) \in T_{P} Q$ onto $p \in P$.

6. $\epsilon_{1}: T_{P}^{*} Q \rightarrow T^{*} Q$ is the projection that maps $\left(\alpha_{q}, p\right) \in T_{P}^{*} Q$ onto $\alpha_{q} \in T^{*} Q$.

7. $\epsilon_{2}: T_{P}^{*} Q \rightarrow P$ is the projection that maps $\left(\alpha_{q}, p\right) \in T_{P}^{*} Q$ onto $p \in P$.

8. The Legendre transform $\mathbb{F} L: T_{P} Q \rightarrow T_{P}^{*} Q$ maps $\left(v_{q}, p\right) \in T_{P} Q$ to $\left(\alpha_{q}, p\right) \in T_{P}^{*} Q$ where $\alpha_{q} \in T_{q}^{*} Q$ is determined from

$$
\left\langle\alpha_{q}, w_{q}\right\rangle=\left.\frac{d}{d u}\right|_{u=0} L\left(v_{q}+u w_{q}, p\right),
$$

for arbitrary $w_{q} \in T_{q} Q$.

9. The energy $E_{L}$ is a function on $T_{P} Q$ defined by $E_{L}\left(v_{q}, p\right)=\left\langle\mathbb{F} L\left(v_{q}, p\right),\left(v_{q}, p\right)\right\rangle-L\left(v_{q}, p\right)$. (Here the contraction of an element $\left(\alpha_{q}, p\right) \in T_{P}^{*} Q$ with $\left(v_{q}, p\right) \in T_{P} Q$ is defined naturally as $\left\langle\left(\alpha_{q}, p\right),\left(v_{q}, p\right)\right\rangle=\left\langle\alpha_{q}, v_{q}\right\rangle$.)

10. By means of the Legendre transform we can pull-back the presymplectic 2-form $\epsilon_{1}^{*} \omega_{Q}+\epsilon_{2}^{*} \mathcal{B}$ on $T_{P}^{*} Q$ to a presymplectic 2-form $\mathbb{F} L^{*}\left(\epsilon_{1}^{*} \omega_{Q}+\epsilon_{2}^{*} \mathcal{B}\right)$ on $T_{P} Q$. The latter is denoted by $\Omega^{L, \mathcal{B}}$ (Here $\omega_{Q}=d \theta_{Q}$, with $\theta_{Q}$ the Poincaré-Cartan 1-form on $T^{*} Q$ and a presymplectic 2 -form is understood to be a closed 2 -form, not necessarily of constant rank.)

Proposition 2. Given a curve $p(t)$ in $P$, and let $\gamma(t)$ denote the curve in $T_{P} Q$ equal to $(\dot{q}(t), p(t)) \in T_{P} Q$ with $q(t)=\epsilon(p(t))$. The curve $p(t)$ in $P$ is a solution to the Euler-Lagrange equations for the magnetic Lagrangian system $(\epsilon: P \rightarrow Q, L, \mathcal{B})$ iff $\gamma(t)$ is a solution to the presymplectic equation

$$
i_{\dot{\gamma}(t)} \Omega^{L, \mathcal{B}}(\gamma(t))=-d E_{L}(\gamma(t)) .
$$

Locally, the presymplectic equation coincides with the previously mentioned Euler-Lagrange equations, since

$$
\begin{aligned}
& \Omega^{L, \mathcal{B}}=d\left(\frac{\partial L}{\partial v^{i}}\right) \wedge d q^{i}+\frac{1}{2} \mathcal{B}_{i j} d q^{i} \wedge d q^{j}+\mathcal{B}_{i a} d q^{i} \wedge d p^{a}+\frac{1}{2} \mathcal{B}_{a b} d p^{a} \wedge d p^{b}, \\
& d E_{L}=v^{i} d\left(\frac{\partial L}{\partial v^{i}}\right)+\frac{\partial L}{\partial v^{i}} d v^{i}-d L=v^{i} d\left(\frac{\partial L}{\partial v^{i}}\right)-\frac{\partial L}{\partial q^{i}} d q^{i}-\frac{\partial L}{\partial p^{a}} d p^{a} .
\end{aligned}
$$

Definition 9. A magnetic Lagrangian system $(\epsilon: P \rightarrow Q, L, \mathcal{B})$

1 ) is hyperregular if $\mathbb{F} L$ is a diffeomorphism and if the restriction of $\mathcal{B}$ to $V \epsilon$ is nondegenerate,

$2)$ is of mechanical type if $L\left(v_{q}, p\right)=\frac{1}{2}\left\langle\left\langle\left(v_{q}, p\right),\left(v_{q}, p\right)\right\rangle_{\rho_{1}}-V(p)\right.$ with $\left\langle\langle\cdot, \cdot\rangle_{\rho_{1}}\right.$ is a metric on the vector bundle $\rho_{1}: T_{P} Q \rightarrow P$ and $V$ is a function on $P$.

Note that if $\mathcal{B}$ is nondegenerate, then the typical fibre of $P$ necessarily has to be evendimensional. In a local coordinate chart the nondegeneracy condition on $\mathcal{B}$ is expressed by $\operatorname{det} \mathcal{B}_{a b} \neq 0$. 
Proposition 3. If a magnetic Lagrangian system $(\epsilon: P \rightarrow Q, L, \mathcal{B})$ is hyperregular, the 2-form $\Omega^{L, \mathcal{B}}=\mathbb{F} L^{*}\left(\epsilon_{1}^{*} \omega_{Q}+\epsilon_{2}^{*} \mathcal{B}\right)$ determines a symplectic structure on $T_{P} Q$.

Proof. Assume that the magnetic Lagrangian system is hyperregular. The nondegeneracy of $\Omega^{L, \mathcal{B}}$ is easily checked if we work in a coordinate chart adapted to the fibration:

$$
\Omega^{L, \mathcal{B}}=d\left(\frac{\partial L}{\partial v^{i}}\right) \wedge d q^{i}+\frac{1}{2} \mathcal{B}_{i j} d q^{i} \wedge d q^{j}+\mathcal{B}_{i a} d q^{i} \wedge d p^{a}+\frac{1}{2} \mathcal{B}_{a b} d p^{a} \wedge d p^{b} .
$$

Since $\mathbb{F} L$ is a diffeomorphism, the 1 -forms $d\left(\frac{\partial L}{\partial v^{i}}\right), d q^{i}$ and $d p^{a}$ provide pointwise a basis on $T^{*}\left(T_{P} Q\right)$. One can now use standard arguments to prove the nondegeneracy.

We conclude that a hyperregular magnetic Lagrangian system has a symplectic structure although the Lagrangian itself is singular when interpreted as a function on $T P$. The energy $E_{L}$ is the Hamiltonian. This is an important observation.

Remark 1. Throughout the paper we only consider magnetic Lagrangian systems that are hyperregular. This is not a true restriction. The results remain valid for general magnetic Lagrangian systems: instead of relating Routh reduction to symplectic reduction, it is possible to relate it to presymplectic reduction [7].

Remark 2. We conclude this section with a remark on equivalent magnetic Lagrangian systems. Roughly speaking, two Lagrangian systems are equivalent if the resulting dynamics coincide, more specifically if they produce the same Euler-Lagrange equations. It is well known that for a Lagrangian system the Euler-Lagrange equations do not change when the Lagrangian is augmented with a total time derivative of a function on the configuration space. For magnetic Lagrangian systems this gauge freedom can be extended.

Consider a 1-form $\alpha$ along $\epsilon$, i.e. a section of $\epsilon_{2}: T_{P}^{*} Q \rightarrow P$, or in other words a linear function on $T_{P} Q$ given by $\left(v_{q}, p\right) \mapsto\left\langle\alpha(p),\left(v_{q}, p\right)\right\rangle$. When $\alpha: P \rightarrow T_{P}^{*} Q$ is composed with $T^{*} \epsilon \circ \epsilon_{1}: T_{P}^{*} Q \rightarrow T^{*} P$, it determines a 1-form on $P$ which, with a slight abuse of notation, we denote by $\epsilon^{*} \alpha$. Now consider the function $L^{\prime}\left(v_{q}, p\right)=L\left(v_{q}, p\right)-\left\langle\alpha(p),\left(v_{q}, p\right)\right\rangle$ and the gyroscopic 2-form $\mathcal{B}^{\prime}=\mathcal{B}+d \epsilon^{*} \alpha$. Together they define a new magnetic Lagrangian system $\left(\epsilon: P \rightarrow Q, L^{\prime}, \mathcal{B}^{\prime}\right)$ which is equivalent to $(\epsilon: P \rightarrow Q, L, \mathcal{B})$.

Lemma 1. The magnetic Lagrangian systems $\left(\epsilon, L^{\prime}, \mathcal{B}^{\prime}\right)$ and $(\epsilon, L, \mathcal{B})$ are equivalent.

This is a reformulation of a well-known result in classical mechanics, saying that an exact gyroscopic force can be taken into account by means of a velocity dependent potential [19].

Proof. We show that any solutions to the Euler-Lagrange equations of $\left(\epsilon, L^{\prime}, \mathcal{B}^{\prime}\right)$ is a solution to the Euler-Lagrange equations of $(\epsilon, L, \mathcal{B})$. We work in a local coordinate neighborhood $\left(q^{i}, p^{a}\right)$ as before. We fix a coordinate expression for $\alpha=\alpha_{i}\left(q^{j}, p^{a}\right) d q^{i}$. The Euler-Lagrange equations for $\left(\epsilon, L^{\prime}, \mathcal{B}^{\prime}\right)$ equal, with $\dot{q}^{i}=v^{i}$ and $\dot{p}^{a}=v^{a}$

$$
\begin{aligned}
& \frac{d}{d t}\left(\frac{\partial L^{\prime}}{\partial v^{i}}\right)-\frac{\partial L^{\prime}}{\partial q^{i}}=\mathcal{B}_{i j}^{\prime} v^{j}+\mathcal{B}_{i a}^{\prime} v^{a}, \quad i=1, \ldots, n, \\
& -\frac{\partial L^{\prime}}{\partial p^{a}}=-\mathcal{B}_{i a}^{\prime} v^{i}+\mathcal{B}_{a b}^{\prime} v^{b}, \quad a=1, \ldots, k .
\end{aligned}
$$

It now remains to substitute the definition of $L^{\prime}$ and $\mathcal{B}^{\prime}$, i.e. $L^{\prime}=L-\alpha_{i} v^{i}$ and

$$
\mathcal{B}_{i j}^{\prime}=\mathcal{B}_{i j}+\left(\frac{\partial \alpha_{j}}{\partial q^{i}}-\frac{\partial \alpha_{i}}{\partial q^{j}}\right), \quad \mathcal{B}_{i a}^{\prime}=\mathcal{B}_{i a}-\frac{\partial \alpha_{i}}{\partial p^{a}}, \quad \mathcal{B}_{a b}^{\prime}=\mathcal{B}_{a b}
$$




\section{Magnetic Lagrangian systems with symmetry}

\subsection{Symplectic reduction: a brief introduction}

An overall reference for this section is e.g. [12]. Let $(M, \omega)$ be a symplectic manifold on which $G$ acts on the right, $\Psi^{M}: M \times G \rightarrow M$. Given a function $f: M \rightarrow \mathfrak{g}^{*}$, then $f_{\xi}$ for $\xi \in \mathfrak{g}$ denotes the real valued function on $M$ defined by $f_{\xi}(m)=\langle f(m), \xi\rangle$.

\section{Definition 10.}

1. The action $\Psi^{M}$ is said to be canonical if $\left(\Psi_{g}^{M}\right)^{*} \omega=\omega$ for all $g \in G$.

2. A map $J: M \rightarrow \mathfrak{g}^{*}$ is a momentum map if $i_{\xi_{M}} \omega=-d J_{\xi}$, for $\xi \in \mathfrak{g}$ arbitrary.

3. If $M$ is connected, the non-equivariance 1-cocycle $\sigma$ of the momentum map $J$ equals

$$
\sigma: G \rightarrow \mathfrak{g}^{*}: g \mapsto J\left(m g^{-1}\right)-\operatorname{Ad}_{g^{-1}}^{*}(J(m))
$$

where $m$ is arbitrary in $M$.

The definition of $\sigma$ is independent of $m$ (see [1]). Recall that a 1-cocycle with values in $\mathfrak{g}^{*}$ statisfies, for $g, h \in G$ arbitrary,

$$
\sigma(g h)=\sigma(g)+\operatorname{Ad}_{g^{-1}}^{*} \sigma(h) .
$$

Definition 11. Let $\sigma$ be a 1-cocycle with values in $\mathfrak{g}^{*}$. The affine action of $G$ on $\mathfrak{g}^{*}$ with 1-cocycle $\sigma$ is given by $(g, \mu) \mapsto \operatorname{Ad}_{g}^{*} \mu+\sigma\left(g^{-1}\right)$ for arbitrary $\mu \in \mathfrak{g}^{*}$ and $g \in G$.

The momentum map $J$ is equivariant with respect to the affine action with 1-cocyle from Definition 10: $J(m g)=\operatorname{Ad}_{g}^{*} J(m)+\sigma\left(g^{-1}\right)$. For a fixed element $\mu \in \mathfrak{g}^{*}$, the Lie group $G_{\mu}<G$ denotes the isotropy subgroup of $\mu$ w.r.t. the affine action, i.e. $g \in G_{\mu}$ if $\mu=\operatorname{Ad}_{g}^{*} \mu+\sigma\left(g^{-1}\right)$ or equivalently if

$$
\mu-J(m g)=\operatorname{Ad}_{g}^{*}(\mu-J(m))
$$

Theorem 2 (symplectic reduction SR). Let $(M, \omega)$ be a symplectic manifold, with $G$ acting canonically on $M$. Let $J$ be a momentum map for this action with non-equivariance cocycle $\sigma$. Assume that $\mu$ is a regular value of $J$, and denote by $G_{\mu}$ the isotropy group of $\mu$ under the affine action of $G$ on $\mathfrak{g}^{*}$. Then the pair $\left(M_{\mu}, \omega_{\mu}\right)$ is a symplectic manifold, with $M_{\mu}=J^{-1}(\mu) / G_{\mu}$ and with $\omega_{\mu}$ a 2-form on $M_{\mu}$ uniquely determined from $i_{\mu}^{*} \omega=\pi_{\mu}^{*} \omega_{\mu}$, with $i_{\mu}: J^{-1}(\mu) \rightarrow M$ and $\pi_{\mu}: J^{-1}(\mu) \rightarrow M_{\mu}=J^{-1}(\mu) / G_{\mu}$.

Any Hamiltonian $h$ on $M$ which is invariant under the action of $G$ induces a function $h_{\mu}$ on $M_{\mu}$ satisfying $\pi_{\mu}^{*} h_{\mu}=i_{\mu}^{*} h$. The Hamiltonian vector field $X_{h}$ is tangent to $J^{-1}(\mu)$ and the corresponding vector field on $J^{-1}(\mu)$ is $\pi_{\mu}$-related to the Hamiltonian vector field $X_{h_{\mu}}$ on $M_{\mu}$.

\subsection{Invariant magnetic Lagrangian systems and momentum maps}

In order to define invariant magnetic Lagrangian systems, we start from a free and proper action of a Lie group $G$ on both $P$ and $Q$ such that they commute with $\epsilon$ : for arbitrary $g \in G$,

$$
\epsilon \circ \Psi_{g}^{P}=\Psi_{g}^{Q} \circ \epsilon .
$$

In other words, $G$ acts on $\epsilon: P \rightarrow Q$ by bundle automorphisms. The projections of the principal bundles are denoted by $\pi^{Q}: Q \rightarrow Q / G$ and $\pi^{P}: P \rightarrow P / G$. These actions induce lifted right actions on $T_{P} Q$ and $T_{P}^{*} Q$ : 
1) $\Psi_{g}^{T_{P} Q}\left(v_{q}, p\right):=\left(T \Psi_{g}^{Q}\left(v_{q}\right), \Psi_{g}^{P}(p)\right)$ for $\left(v_{q}, p\right) \in T_{P} Q$ and $g \in G$,

2) $\Psi_{g}^{T_{P}^{*} Q}\left(\alpha_{q}, p\right):=\left(T^{*} \Psi_{g^{-1}}^{Q}\left(\alpha_{q}\right), \Psi_{g}^{P}(p)\right)$ for $\left(\alpha_{q}, p\right) \in T_{P}^{*} Q$ and $g \in G$.

Definition 12. A magnetic Lagrangian system $(\epsilon: P \rightarrow Q, L, \mathcal{B})$ is $G$-invariant if

1) $\mathcal{B}$ is invariant under the action of $\Psi^{P}:\left(\Psi_{g}^{P}\right)^{*} \mathcal{B}=\mathcal{B}$ for all $g \in G$,

2) the Lagrangian $L$ is invariant under the lifted action $\Psi^{T_{P} Q}$ on $T_{P} Q$, i.e. $L \circ \Psi_{g}^{T_{P} Q}=L$ for all $g \in G$.

Let $(\epsilon: P \rightarrow Q, L, \mathcal{B})$ be a $G$-invariant hyperregular magnetic Lagrangian system and consider the symplectic 2 -form $\epsilon_{1}^{*} \omega_{Q}+\epsilon_{2}^{*} \mathcal{B}$ on $T_{P}^{*} Q$.

Proposition 4. The action $\Psi^{T_{P}^{*} Q}$ is canonical and the Legendre transform $\mathbb{F} L: T_{P} Q \rightarrow T_{P}^{*} Q$ is equivariant, i.e. $\mathbb{F} L \circ \Psi_{g}^{T_{P} Q}=\Psi_{g}^{T_{P}^{*} Q} \circ \mathbb{F} L$. As a consequence the action $\Psi^{T_{P} Q}$ is canonical w.r.t. $\Omega^{L, \mathcal{B}}$.

Proof. Note that $\epsilon_{1} \circ \Psi_{g}^{T_{P}^{*} Q}=T^{*} \Psi_{g^{-1}}^{Q} \circ \epsilon_{1}$ and $\epsilon_{2} \circ \Psi_{g}^{T_{P}^{*} Q}=\Psi_{g}^{P} \circ \epsilon_{2}$. Since the action $\Psi_{g}^{T^{*} Q}=$ $T^{*} \Psi_{g^{-1}}^{Q}$ on $T^{*} Q$ is canonical w.r.t. $\omega_{Q}$ and since $\mathcal{B}$ is a $G$-invariant 2 -form on $P$, we conclude that $\Psi_{P}^{T_{P}^{*}}$ is canonical. To show the equivariance of $\mathbb{F} L$ we use its definition: let $g \in G, v_{q}, w_{q} \in T Q$ be arbitrary, then

$$
\begin{aligned}
\left\langle\mathbb{F} L\left(\Psi_{g}^{T_{P} Q}\left(v_{q}, p\right)\right), \Psi_{g}^{T_{P} Q}\left(w_{q}, p\right)\right\rangle & =\left.\frac{d}{d u}\right|_{u=0} L\left(\Psi_{g}^{T_{P} Q}\left(v_{q}+u w_{q}\right)\right) \\
& =\left.\frac{d}{d u}\right|_{u=0} L\left(v_{q}+u w_{q}\right)=\left\langle\mathbb{F} L\left(v_{q}, p\right),\left(w_{q}, p\right)\right\rangle .
\end{aligned}
$$

In order to define a momentum map, we introduce a concept that is similar to the so-called $\mathcal{B g}$-potential in [12].

Definition 13. A $\mathfrak{g}^{*}$-valued function $\delta$ on $P$ is a $\mathcal{B g}$-potential for the magnetic 2 -form $\mathcal{B}$ if $i_{\xi_{P}} \mathcal{B}=d \delta_{\xi}$ for any $\xi \in \mathfrak{g}$.

Recall that $\delta_{\xi}$ is defined pointwise as $\delta_{\xi}(p)=\langle\delta(p), \xi\rangle$. If $\mathcal{B}$ is $G$-invariant and admits a $\mathcal{B} \mathfrak{g}$-potential $\delta$, then it follows that $d\left(\left(\Psi_{g}^{P}\right)^{*} \delta_{\xi}-\delta_{\operatorname{Ad}_{g} \xi}\right)=0$. If $P$ is connected, this induces a $\mathfrak{g}^{*}$-valued 1-cocycle on $G$ :

$$
\sigma_{\delta}: G \rightarrow \mathfrak{g}^{*} ; \quad g \mapsto\left(\Psi_{g^{-1}}^{P}\right)^{*} \delta(p)-\mathrm{Ad}_{g^{-1}}^{*} \cdot \delta(p),
$$

with $p$ arbitrary in $P$. The infinitesimal version of this 1-cocycle is denoted by $\Sigma_{\delta}(\xi, \eta)=$ $-\left\langle T_{e} \sigma_{\delta}(\xi), \eta\right\rangle$. It is a 2-cocycle on the Lie algebra and satisfies $\Sigma_{\delta}(\xi, \eta)=-\mathcal{B}\left(\xi_{P}, \eta_{P}\right)-\delta_{[\xi, \eta]}=$ $\xi_{P}\left(\delta_{\eta}\right)-\delta_{[\xi, \eta]}$. For the following proposition, recall from Definition 2 that $\left(\psi^{Q}\right)^{*}: T^{*} Q \rightarrow \mathfrak{g}^{*}$ is the dual of the infinitesimal action of $G$ on $Q$.

Proposition 5. The map $J_{\delta}=\left(\psi^{Q}\right)^{*} \circ \epsilon_{1}-\delta \circ \epsilon_{2}$ is a momentum map for the symplectic manifold $\left(T_{P}^{*} Q, \epsilon_{1}^{*} \omega_{Q}+\epsilon_{2}^{*} \mathcal{B}\right)$ with non-equivariance 1 -cocycle $-\sigma_{\delta}$. Due to the equivariance of $\mathbb{F} L$, the map $J_{L, \delta}=J_{\delta} \circ \mathbb{F} L$ is a momentum map for the symplectic manifold $\left(T_{P} Q, \Omega^{L, \mathcal{B}}\right)$ with nonequivariance 1-cocycle $-\sigma_{\delta}$.

Proof. The map $\left(\psi^{Q}\right)^{*}: T^{*} Q \rightarrow \mathfrak{g}^{*}$ is an equivariant momentum map for the symplectic manifold $\left(T^{*} Q, \omega_{Q}\right)$. It is straightforward that the combined map $J_{\delta}=\left(\psi^{Q}\right)^{*} \circ \epsilon_{1}-\delta \circ \epsilon_{2}$ is a momentum map for the lifted action on $T_{P}^{*} Q$ w.r.t. the symplectic form $\epsilon_{1}^{*} \omega_{Q}+\epsilon_{2}^{*} \mathcal{B}$ with non-equivariance cocycle $-\sigma_{\delta}$. The rest of the statement follows by construction.

We conclude that the symplectic structures $\left(T_{P} Q, \Omega^{L, \mathcal{B}}\right)$ and $\left(T_{P}^{*} Q, \epsilon_{1}^{*} \omega_{Q}+\epsilon_{2}^{*} \mathcal{B}\right)$ associated to a $G$-invariant and hyperregular magnetic Lagrangian system with a $\mathcal{B g}$-potential $\delta$ admit a momentum map with cocycle $-\sigma_{\delta}$ and are amenable to symplectic reduction. 


\section{Routh reduction for magnetic Lagrangian systems}

\subsection{Magnetic cotangent bundle reduction}

In this section we study the reduction of the symplectic manifold $\left(T_{P}^{*} Q, \epsilon_{1}^{*} \omega_{Q}+\epsilon_{2}^{*} \mathcal{B}\right)$ determined from the magnetic 2 -form $\mathcal{B}$ of a $G$-invariant hyperregular magnetic Lagrangian system with $\mathcal{B g}$-potential $\delta$. From Proposition 5, it follows that the function $J_{\delta}$ is a momentum map with non-equivariance cocycle $-\sigma_{\delta}$. As usual, $G_{\mu}$ denotes the isotropy subgroup of $\mu \in \mathfrak{g}^{*}$ for the affine action.

Proposition 6. Fix a connection $\mathcal{A}^{Q}$ on the bundle $\pi^{Q}: Q \rightarrow Q / G$ and a regular value $\mu$ of the momentum map $J_{\delta}$.

1. $g \in G_{\mu}$ iff $\mu+\delta(p g)=\operatorname{Ad}_{g}^{*}(\mu+\delta(p))$.

2. $\mathcal{A}^{P}=\epsilon^{*} \mathcal{A}^{Q}$ is a principal connection on $\pi^{P}: P \rightarrow P / G$.

3. The 1-form $\left\langle\mu+\delta, \mathcal{A}^{P}\right\rangle$ on $P$ is $G_{\mu}$-invariant.

4. The 2 -form $\mathcal{B}+d\left(\left\langle\mu+\delta, \mathcal{A}^{P}\right\rangle\right)$ is $G_{\mu}$-invariant and reducible to a 2 -form on $P / G_{\mu}$.

5. The quotient manifold $J_{\delta}^{-1}(\mu) / G_{\mu}$ is diffeomorphic to $T_{P / G_{\mu}}^{*}(Q / G)$.

Proof. Recall the pointwise definition of $\left\langle\mu+\delta, \mathcal{A}^{P}\right\rangle$ : given $v_{p} \in T P$, then $\left\langle\mu+\delta, \mathcal{A}^{P}\right\rangle(p)\left(v_{p}\right):=$ $\left\langle\mu+\delta(p), \mathcal{A}^{P}(p)\left(v_{p}\right)\right\rangle$. Below, $\left\langle\mu+\delta(p), \mathcal{A}^{P}(p)\right\rangle \in T_{p}^{*} P$ denotes the cotangent vector $\langle\mu+$ $\left.\delta, \mathcal{A}^{P}\right\rangle(p)$. We continue with the proof.

1. This is straightforward from the definition of $\sigma_{\delta}$.

2. The $\mathfrak{g}^{*}$-valued 1 -form $\mathcal{A}^{P}$ determines a principal connection if it satisfies $\mathcal{A}^{P}\left(\xi_{P}\right)=\xi$ for all $\xi \in \mathfrak{g}$, and if $\left(\Psi_{g}^{P}\right)^{*} \mathcal{A}^{P}=\operatorname{Ad}_{g^{-1}} \cdot \mathcal{A}^{P}$ for all $g \in G$ :

$$
\begin{aligned}
& \mathcal{A}^{P}\left(\xi_{P}\right)(p)=\mathcal{A}^{Q}(\epsilon(p))\left(T \epsilon\left(\xi_{P}(p)\right)\right)=\mathcal{A}^{Q}(\epsilon(p))\left(\xi_{Q}(\epsilon(p))\right)=\xi \quad \text { and } \\
& \left(\Psi_{g}^{P}\right)^{*} \mathcal{A}^{P}=\left(\Psi_{g}^{P}\right)^{*} \epsilon^{*} \mathcal{A}^{Q}=\epsilon^{*}\left(\Psi_{g}^{Q}\right)^{*} \mathcal{A}^{Q}=\operatorname{Ad}_{g^{-1}} \cdot \epsilon^{*} \mathcal{A}^{Q}=\operatorname{Ad}_{g^{-1}} \cdot \mathcal{A}^{P} .
\end{aligned}
$$

3. The pull-back 1-form $\left(\Psi_{g}^{P}\right)^{*}\left\langle\mu+\delta, \mathcal{A}^{P}\right\rangle$ equals

$$
\left\langle\mu+\delta(p g),\left(\left(\Psi_{g}^{P}\right)^{*} \mathcal{A}^{P}\right)(p)\right\rangle=\left\langle\operatorname{Ad}_{g}^{*}(\mu+\delta(p)), \operatorname{Ad}_{g^{-1}} \cdot \mathcal{A}^{P}(p)\right\rangle .
$$

4. That $\mathcal{B}+d\left(\left\langle\mu+\delta, \mathcal{A}^{P}\right\rangle\right)$ is $G_{\mu}$-invariant is a straightforward consequence of 3 . It is projectable to $P / G_{\mu}$ since the contraction with any fundamental vector field $\xi_{P}$ with $\xi \in \mathfrak{g}_{\mu}$ vanishes:

$$
\begin{aligned}
i_{\xi_{P}}\left(d\left(\left\langle\mu+\delta, \mathcal{A}^{P}\right\rangle\right)+\mathcal{B}\right) & =\mathcal{L}_{\xi_{P}}\left(\left\langle\mu+\delta, \mathcal{A}^{P}\right\rangle\right)-d(\langle\mu+\delta, \xi\rangle)+d \delta_{\xi} \\
& =\left\langle\xi_{P}(\mu+\delta), \mathcal{A}^{P}\right\rangle+\left\langle\mu+\delta, \operatorname{ad}_{-\xi} \cdot \mathcal{A}^{P}\right\rangle-d \delta_{\xi}+d \delta_{\xi} \\
& =\left\langle\xi_{P}(\delta)-\operatorname{ad}_{\xi}^{*} \mu-\operatorname{ad}_{\xi}^{*} \delta, \mathcal{A}^{P}\right\rangle=0,
\end{aligned}
$$

where we used the fact that $\xi \in \mathfrak{g}_{\mu}$, or equivalently $\operatorname{ad}_{\xi}^{*} \mu=i_{\xi} \Sigma_{\delta}$.

5. Similar to cotangent bundle reduction, we use a 'shift map' to construct the required diffeomorphism. Recall that $V \pi^{Q}$ denotes the bundle of tangent vectors vertical to $\pi^{Q}: Q \rightarrow$ $Q / G$. The subbundle $V^{0} \pi^{Q}$ of $T^{*} Q$ is defined as the annihilator of $V \pi^{Q}$. Below we introduce a shift map $\phi_{\mu}^{\mathcal{A}}$ between $J_{\delta}^{-1}(\mu)$ and $V_{P}^{0} \pi^{Q}=V^{0} \pi^{Q} \times{ }_{Q} P$ and we show that it is equivariant w.r.t. to actions of $G_{\mu}$ obtained by restriction of $\Psi^{T_{P} Q}$ to $J_{\delta}^{-1}(\mu)$ and $V_{P}^{0} \pi^{Q}$. This is sufficient for $\phi_{\mu}^{\mathcal{A}}$ to project to a diffeomorphism $\left[\phi_{\mu}^{\mathcal{A}}\right]$ between the quotient spaces. This provides us with the desired diffeomorphism because the quotient of $V_{P}^{0} \pi^{Q}$ is well known: $V_{P}^{0} \pi^{Q} / G_{\mu}=T_{P / G_{\mu}}^{*}(Q / G)$. 
Definition of the shift map. In the following we use a slight abuse of notations: if we write $\left\langle\mu+\delta(p), \mathcal{A}^{Q}(q)\right\rangle$ for some $p \in P$ and $q=\epsilon(p)$, then this is the cotangent vector in $T_{q}^{*} Q$ determined by $v_{q} \mapsto\left\langle\mu+\delta(p), \mathcal{A}^{Q}(q)\left(v_{q}\right)\right\rangle$. Let $\phi_{\mu}^{\mathcal{A}}$ be the map

$$
\phi_{\mu}^{\mathcal{A}}: J_{\delta}^{-1}(\mu) \rightarrow V_{P}^{0} \pi_{Q} ; \quad\left(\alpha_{q}, p\right) \mapsto\left(\alpha_{q}-\left\langle\mu+\delta(p), \mathcal{A}^{Q}(q)\right\rangle, p\right) .
$$

It is well defined, i.e. $\phi_{\mu}^{\mathcal{A}}\left(\alpha_{q}, p\right) \in V_{P}^{0} \pi^{Q}$ for any $\left(\alpha_{q}, p\right)$ in the level set $J_{\delta}^{-1}(\mu)$ since

$$
\begin{aligned}
\left\langle\phi_{\mu}^{\mathcal{A}}\left(\alpha_{q}, p\right),\left(\xi_{Q}(q), p\right)\right\rangle & =\left\langle\alpha_{q}, \xi_{Q}(q)\right\rangle-\left\langle\mu+\delta(p), \mathcal{A}^{Q}(q)\left(\xi_{Q}(q)\right)\right\rangle \\
& =\left\langle J_{\delta}\left(\alpha_{q}, p\right), \xi\right\rangle-\langle\mu, \xi\rangle=0 .
\end{aligned}
$$

Equivariance of the shift map. For arbitrary $g \in G_{\mu}$,

$$
\begin{aligned}
\phi_{\mu}^{\mathcal{A}}\left(\Psi_{g}^{T_{P}^{*} Q}\left(\alpha_{q}, p\right)\right) & =\left(T^{*} \Psi_{g^{-1}}^{Q}\left(\alpha_{q}\right)-\left\langle\mu+\delta(p g), \mathcal{A}^{Q}(q g)\right\rangle, p g\right) \\
& =\left(T^{*} \Psi_{g^{-1}}^{Q}\left(\alpha_{q}-\left\langle\mu+\delta(p g), \operatorname{Ad}_{g^{-1}} \cdot \mathcal{A}^{Q}(q)\right\rangle\right), p g\right) \\
& =\left(T^{*} \Psi_{g^{-1}}^{Q}\left(\alpha_{q}-\left\langle\mu+\delta(p), \mathcal{A}^{Q}(q)\right\rangle\right), p g\right)=\Psi_{g}^{T_{P}^{*} Q}\left(\phi_{\mu}^{\mathcal{A}}\left(\alpha_{q}, p\right)\right) .
\end{aligned}
$$

The projection of $\phi_{\mu}^{\mathcal{A}}$ is thus well defined, and it is denoted by $\left[\phi_{\mu}^{\mathcal{A}}\right]$, i.e. $\left[\phi_{\mu}^{\mathcal{A}}\right]: J_{\delta}^{-1}(\mu) / G_{\mu} \rightarrow$ $V_{P}^{0} \pi^{Q} / G_{\mu}=T_{P / G_{\mu}}^{*}(Q / G)$.

Definition 14. $\tilde{\mathcal{B}}$ is the 2 -form on $P / G_{\mu}$ obtained after reducing $\mathcal{B}+d\left(\left\langle\mu+\delta, \mathcal{A}^{P}\right\rangle\right)$.

In the following proposition we put a symplectic structure on the fibred product $T_{P / G_{\mu}}^{*}(Q / G)$ $=T^{*}(Q / G) \times_{Q / G} P / G_{\mu}$. The notations are similar to those on $T_{P}^{*} Q$.

Definition 15. We define the projections

1) $\tilde{\epsilon}_{1}: T_{P / G_{\mu}}^{*}(Q / G) \rightarrow T^{*}(Q / G)$,

2) $\tilde{\epsilon}_{2}: T_{P / G_{\mu}}^{*}(Q / G) \rightarrow P / G_{\mu}$.

The canonical symplectic 2 -form on $T^{*}(Q / G)$ is denoted by $\omega_{Q / G}$.

Completely analogous to the construction on $T_{P}^{*} Q$, we introduce $\tilde{\epsilon}_{1}^{*} \omega_{Q / G}+\epsilon_{2}^{*} \tilde{\mathcal{B}}$ as a 2 -form on $T_{P / G_{\mu}}^{*}(Q / G)$.

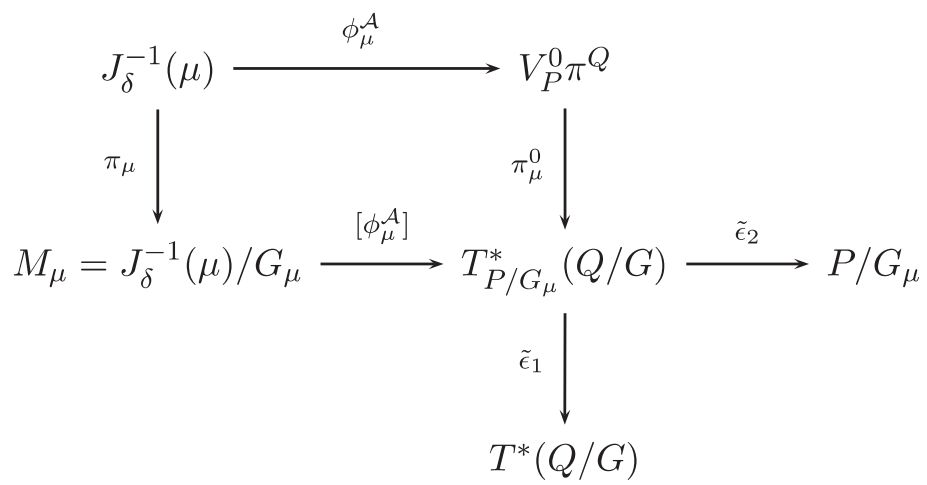

Figure 1. Cotangent bundle reduction (notations as in Theorem 2 with $M=T_{P}^{*} Q$ ). 
Theorem 3 (Generalized magnetic cotangent bundle reduction). Let $\mu$ denote a regular value of the momentum map $J_{\delta}$ for the symplectic structure $\left(T_{P}^{*} Q, \epsilon_{1}^{*} \omega_{Q}+\epsilon_{2}^{*} \mathcal{B}\right)$ with isotropy subgroup $G_{\mu}$, and let $\mathcal{A}^{Q}$ be a principal connection on $Q \rightarrow Q / G$. Then the symplectically reduced manifold $\left(M_{\mu}, \omega_{\mu}\right)$ is symplectomorphic to $\left.\left(T_{P / G_{\mu}}^{*}(Q / G), \tilde{\epsilon}_{1}^{*} \omega_{Q / G}+\tilde{\epsilon}_{2}^{*} \tilde{\mathcal{B}}\right)\right)$, with symplectomorphism $\left[\phi_{\mu}^{\mathcal{A}}\right]$.

Although this result is a straightforward extension of standard magnetic cotangent bundle reduction in [12], we include a proof because its method turns out to be useful later on.

Proof. Let $i_{0}$ denote the injection $V_{P}^{0} \pi^{Q} \rightarrow T_{P}^{*} Q$ and $\pi_{\mu}^{0}$ the projection $V_{P}^{0} \pi^{Q} \rightarrow T_{P / G_{\mu}}^{*}(Q / G)$. We know that $\left[\phi_{\mu}^{\mathcal{A}}\right]$ is a diffeomorphism, and therefore it only remains to show that the 2 -form $\tilde{\epsilon}_{1}^{*} \omega_{Q / G}+\tilde{\epsilon}_{2}^{*} \tilde{\mathcal{B}}$ is pull-backed to $\omega_{\mu}$ under this map (and therefore, implicitly $\tilde{\epsilon}_{1}^{*} \omega_{Q / G}+\tilde{\epsilon}_{2}^{*} \tilde{\mathcal{B}}$ will be nondegenerate).

We use the fact that $\omega_{\mu}$ is uniquely determined by $i_{\mu}^{*} \omega_{Q}=\pi_{\mu}^{*} \omega_{\mu}$, with $i_{\mu}: J_{\delta}^{-1}(\mu) \rightarrow T_{P}^{*} Q$ the natural inclusion and $\pi_{\mu}: J_{\delta}^{-1}(\mu) \rightarrow M_{\mu}$ the projection to the quotient space. Due to the uniqueness property, it is therefore sufficient to show that

$$
\pi_{\mu}^{*}\left(\left[\phi_{\mu}^{\mathcal{A}}\right]^{*}\left(\tilde{\epsilon}_{1}^{*} \omega_{Q / G}+\tilde{\epsilon}_{2}^{*} \tilde{\mathcal{B}}\right)\right)=i_{\mu}^{*}\left(\epsilon_{1}^{*} \omega_{Q}+\epsilon_{2}^{*} \mathcal{B}\right) .
$$

The left-hand side of (2). Since $\left[\phi_{\mu}^{\mathcal{A}}\right] \circ \pi_{\mu}=\pi_{\mu}^{0} \circ \phi_{\mu}^{\mathcal{A}}$ the left-hand side of the above equation is $\left(\pi_{\mu}^{0} \circ \phi_{\mu}^{\mathcal{A}}\right)^{*}\left(\tilde{\epsilon}_{1}^{*} \omega_{Q / G}+\tilde{\epsilon}_{2}^{*} \tilde{\mathcal{B}}\right)$.

The right-hand side of (2). We first show the equality of two 1-forms:

$$
i_{\mu}^{*}\left(\epsilon_{1}^{*} \theta_{Q}\right) \quad \text { and } \quad\left(\phi_{\mu}^{\mathcal{A}}\right)^{*}\left(i_{0}^{*}\left(\epsilon_{1}^{*} \theta_{Q}+\epsilon_{2}^{*}\left\langle\mu+\delta, \mathcal{A}^{P}\right\rangle\right)\right) .
$$

Let $\left(\alpha_{q}, p\right) \in J_{\delta}^{-1}(\mu), V_{\left(\alpha_{q}, p\right)} \in T\left(T_{P}^{*} Q\right)$ a tangent vector to $J_{\delta}^{-1}(\mu)$ and $v_{q} \in T_{q} Q$ denotes the projection of $V_{\left(\alpha_{q}, p\right)}$. Then, the first 1-form equals

$$
i_{\mu}^{*}\left(\epsilon_{1}^{*} \theta_{Q}\right)\left(\alpha_{q}, p\right)\left(V_{\left(\alpha_{q}, p\right)}\right)=\theta_{Q}\left(\alpha_{q}\right)\left(T \epsilon_{1}\left(V_{\left(\alpha_{q}, p\right)}\right)\right)=\left\langle\alpha_{q}, v_{q}\right\rangle .
$$

The second 1-form evaluated on this tangent vector gives

$$
\begin{aligned}
\left(\phi_{\mu}^{\mathcal{A}}\right)^{*} & \left(i_{0}^{*}\left(\epsilon_{1}^{*} \theta_{Q}+\epsilon_{2}^{*}\left\langle\mu+\delta, \mathcal{A}^{P}\right\rangle\right)\right)\left(\alpha_{q}, p\right)\left(V_{\left(\alpha_{q}, p\right)}\right) \\
& =\theta_{Q}\left(\alpha_{q}-\left\langle\mu+\delta(p), \mathcal{A}^{Q}(q)\right\rangle\right)\left(T \epsilon_{1}\left(T\left(\phi_{\mu}^{\mathcal{A}}\right)\left(V_{\left(\alpha_{q}, p\right)}\right)\right)\right)+\left\langle\mu+\delta(p), \mathcal{A}^{Q}(q)\left(v_{q}\right)\right\rangle \\
& =\left\langle\alpha_{q}, v_{q}\right\rangle .
\end{aligned}
$$

The two 1-forms are identical and after taking the exterior derivative and adding $\epsilon_{2}^{*} \mathcal{B}$, we obtain that the right-hand side of (2) equals

$$
i_{\mu}^{*}\left(\epsilon_{1}^{*} \omega_{Q}+\epsilon_{2}^{*} \mathcal{B}\right)=\left(\phi_{\mu}^{\mathcal{A}}\right)^{*}\left(i_{0}^{*}\left(\epsilon_{1}^{*} \omega_{Q}+\epsilon_{2}^{*}\left(d\left\langle\mu+\delta, \mathcal{A}^{P}\right\rangle+\mathcal{B}\right)\right)\right) .
$$

Since (i) $\left(\tilde{\epsilon}_{2} \circ \pi_{\mu}^{0}\right)^{*} \tilde{\mathcal{B}}=\left(\epsilon_{2} \circ i_{0}\right)^{*}\left(d\left\langle\mu+\delta, \mathcal{A}^{P}\right\rangle+\mathcal{B}\right)$, and (ii) $\left(\tilde{\epsilon}_{1} \circ \pi_{\mu}^{0}\right)^{*} \theta_{Q / G}=i_{0}^{*} \epsilon_{1}^{*} \theta_{Q}$, one easily verifies that the left-hand side equals the right-hand side in (2).

We conclude with a result on the behavior of a symplectomorphism under symplectic reduction. Assume two symplectic manifolds $(M, \Omega)$ and $\left(M^{\prime}, \Omega^{\prime}\right)$ and a symplectomorphism $f: M \rightarrow M^{\prime}$ (i.e. a map for which $f^{*} \Omega^{\prime}=\Omega$ ) are given. We assume in addition that both $M$ and $M^{\prime}$ are equipped with a canonical free and proper action of $G$. Let $J: M \rightarrow \mathfrak{g}^{*}$ and $J^{\prime}: M^{\prime} \rightarrow \mathfrak{g}^{*}$ denote corresponding momentum maps for these actions on $M$ and $M^{\prime}$ respectively. We say that $f$ is equivariant if $f(m g)=f(m) g$ for arbitrary $m \in P, g \in G$. Note that the non-equivariance cocycles for $J$ and $J^{\prime}$ are equal up to a coboundary. Without loss of generality we may assume that $f^{*} J^{\prime}=J$ and that the non-equivariance cocycles coincide. This in turn guarantees that the affine actions on $\mathfrak{g}^{*}$ coincide and that the isotropy groups of an element $\mu \in \mathfrak{g}^{*}$ coincide for both affine actions. Finally, fix a regular value $\mu \in \mathfrak{g}^{*}$ for both $J$ and $J^{\prime}$. 
Theorem 4. If $f$ is an equivariant symplectic diffeomorphism $M \rightarrow M^{\prime}$, such that $J^{\prime}=J \circ f$, then under symplectic reduction, the symplectic manifolds $\left(M_{\mu}, \Omega_{\mu}\right)$ and $\left(M_{\mu}^{\prime}, \Omega_{\mu}^{\prime}\right)$ are symplectically diffeomorphic under the map

$$
\left[f_{\mu}\right]: M_{\mu} \rightarrow M_{\mu}^{\prime} ; \quad[m]_{G_{\mu}} \mapsto[f(m)]_{G_{\mu}}
$$

Proof. This is a straightforward result. Since $f$ is a diffeomorphism for which $J^{\prime}=J \circ f$, the restriction $f_{\mu}$ of $f$ to $J^{-1}(\mu)$ determines a diffeomorphism from $J^{-1}(\mu)$ to $J^{\prime-1}(\mu)$. The equivariance implies that $f_{\mu}$ reduces to a diffeomorphism $\left[f_{\mu}\right]$ from $M_{\mu}=J^{-1}(\mu) / G_{\mu}$ to $M_{\mu}^{\prime}=$ $J^{\prime-1}(\mu) / G_{\mu}$. It is our purpose to show that $\left[f_{\mu}\right]^{*} \Omega_{\mu}^{\prime}=\Omega_{\mu}$, or since both $\pi_{\mu}$ and $\pi_{\mu}^{\prime}$ are projections, that $\pi_{\mu}^{*} \Omega_{\mu}=f_{\mu}^{*}\left(\pi_{\mu}^{\prime *} \Omega_{\mu}^{\prime}\right)$. The determining property for $\Omega_{\mu}$ is $\pi_{\mu}^{*} \Omega_{\mu}=i_{\mu}^{*} \Omega$ (and similarly for $\left.\Omega_{\mu}^{\prime}\right)$. From diagram chasing we have that $i_{\mu}^{*} \Omega=f_{\mu}^{*}\left(i_{\mu}^{*} \Omega^{\prime}\right)$. Then

$$
\pi_{\mu}^{*} \Omega_{\mu}=i_{\mu}^{*} \Omega=f_{\mu}^{*}\left(i_{\mu}^{\prime *} \Omega^{\prime}\right)=f_{\mu}^{*}\left(\pi_{\mu}^{* *} \Omega_{\mu}^{\prime}\right)=\pi_{\mu}^{*}\left(\left[f_{\mu}\right]^{*} \Omega_{\mu}^{\prime}\right),
$$

since $\pi_{\mu}^{\prime} \circ f_{\mu}=\left[f_{\mu}\right] \circ \pi_{\mu}$ by definition. This concludes the proof.

\subsection{Routh reduction for magnetic Lagrangian systems}

In Proposition 5 we have introduced the momentum map $J_{L, \delta}=J_{\delta} \circ \mathbb{F} L$ for a $G$-invariant hyperregular magnetic Lagrangian system $(\epsilon: P \rightarrow Q, L, \mathcal{B})$, i.e.

$$
\left\langle J_{L, \delta}\left(v_{q}, p\right), \xi\right\rangle=\left\langle\mathbb{F} L\left(v_{q}, p\right),\left(\xi_{Q}(q), p\right)\right\rangle-\delta_{\xi}(p) .
$$

We know from Proposition 4 that the Legendre transform $\mathbb{F} L$ is equivariant. After restriction to $J_{L, \delta}^{-1}(\mu)$ it reduces to a symplectic diffeomorphism between the symplectically reduced spaces

$J_{L, \delta}^{-1}(\mu) / G_{\mu}$ and $J_{\delta}^{-1}(\mu) / G_{\mu}$ (see also Theorem 4). The following diagram summarizes these previous observations.

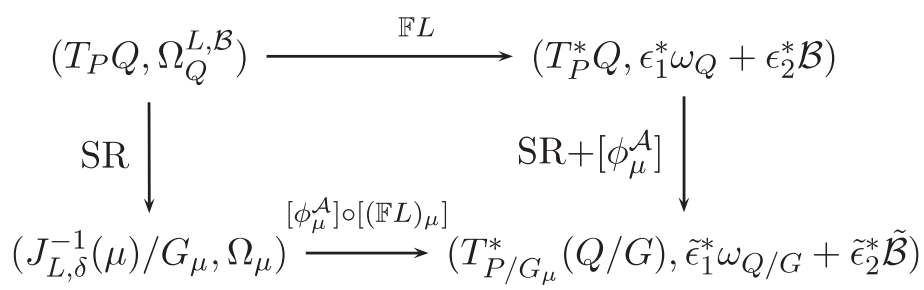

Figure 2. Diagram relating tangent and cotangent reduction $(\mathrm{SR}=$ symplectic reduction).

We are now only three steps away from a description of Routh reduction for magnetic Lagrangian systems. The final goal is to describe the symplectic reduced manifold $\left(J_{L, \delta}^{-1}(\mu) / G_{\mu}, \Omega_{\mu}\right)$ as a symplectic manifold associated with a new magnetic Lagrangian system, with Lagrangian say $L_{\mu}$.

1. The first step is the construction of a diffeomorphism $\Delta_{\mu}: T_{P / G_{\mu}}(Q / G) \rightarrow J_{L, \delta}^{-1}(\mu) / G_{\mu}$.

This is crucial because the Hamiltonian dynamics determined by the Euler-Lagrange equations reduce to Hamiltonian dynamics on a manifold of the form $T_{P / G_{\mu}}(Q / G)$. If we can characterize this reduced dynamics as the Euler-Lagrange equations of a magnetic Lagrangian system with configuration manifold $P / G_{\mu} \rightarrow Q / G$, we have developed a Routh reduction technique for magnetic Lagrangian systems. This characterization consists of the two remaining steps mentioned before: 
2. We show that the composition $\left[\phi_{\mu}^{\mathcal{A}}\right] \circ\left[(\mathbb{F} L)_{\mu}\right] \circ \Delta_{\mu}$ equals the fibre derivative of some Lagrangian function $\tilde{L}$ on $T_{P / G_{\mu}}(Q / G)$, and this implies that the symplectic 2-form $\Delta_{\mu}^{*} \Omega_{\mu}$ on $T_{P / G_{\mu}}(Q / G)$ is of the form

$$
\mathbb{F} \tilde{L}^{*}\left(\tilde{\epsilon}_{1}^{*} \omega_{Q / G}+\tilde{\epsilon}_{2}^{*} \tilde{\mathcal{B}}\right)
$$

which is the symplectic structure associated to the reduced magnetic Lagrangian system $\left(P / G_{\mu} \rightarrow Q / G, \tilde{L}, \tilde{\mathcal{B}}\right)$

3 . We show that the reduction of the energy $E_{L}$ in the symplectic reduction scheme is precisely the energy $E_{\tilde{L}}$ on $T_{P / G_{\mu}}(Q / G)$. This guarantees that the Euler-Lagrange equations associated to the reduced Lagrangian $\tilde{L}$ are related to the Euler-Lagrange equations for $L$.

The new reduced Lagrangian $\tilde{L}$ is what we call the Routhian (it is often alternatively denoted by $R$ or $R^{\mu}$ ). This is summarized in the diagram in Fig. 3.

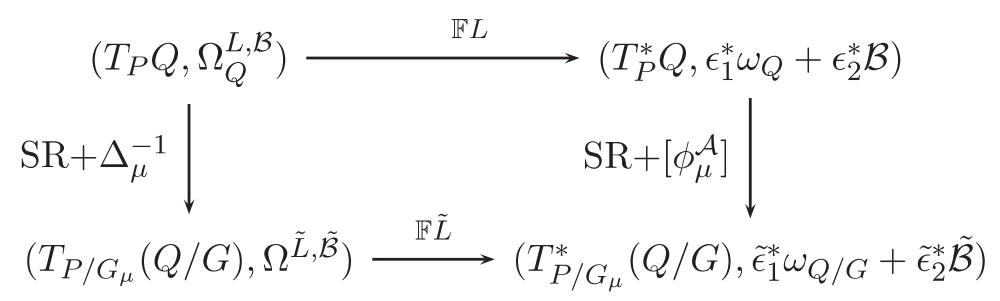

Figure 3. A symplectic view on Routh reduction.

Step 1: the definition of $\Delta_{\mu}: T_{P / G_{\mu}}(Q / G) \rightarrow J_{L, \delta}^{-1}(\mu) / G_{\mu}$. First consider the map $\Pi_{\mu}: J_{L, \delta}^{-1}(\mu) / G_{\mu} \rightarrow T_{P / G_{\mu}}(Q / G)$ which is obtained from the $G_{\mu}$-invariance of the map

$$
J_{L, \delta}^{-1}(\mu) \rightarrow T_{P / G_{\mu}}(Q / G) ;\left(v_{q}, p\right) \mapsto\left(T \pi^{Q}\left(v_{q}\right),[p]_{G_{\mu}}\right) .
$$

We will define the map $\Delta_{\mu}$ as the inverse of $\Pi_{\mu}$. In general however, $\Pi_{\mu}$ is not invertible. The following definition is the analogue of $G$-regularity for a classical Lagrangian.

Definition 16. The Lagrangian $L$ of a $G$-invariant magnetic Lagrangian system is called $G$ regular if the map $\left.J_{L, \delta}\right|_{\left(v_{q}, p\right)}: \mathfrak{g} \rightarrow \mathfrak{g}^{*} ; \xi \mapsto J_{L, \delta}\left(v_{q}+\xi_{Q}(q), p\right)$ is a diffeomorphism for all $\left(v_{q}, p\right) \in T_{P} Q$.

Every mechanical $G$-invariant magnetic Lagrangian system is $G$-regular because $\left.J_{L, \delta}\right|_{\left(v_{q}, p\right)}$ is an affine map modeled on the metric on $\mathfrak{g}$ obtained from the kinetic energy metric.

Proposition 7. $\Pi_{\mu}$ is a diffeomorphism if the Lagrangian is G-regular.

Proof. We construct the inverse for $\Pi_{\mu}$. Choose an element $\left(v_{[q]_{G}},[p]_{G_{\mu}}\right)$ in $T_{P / G_{\mu}}(Q / G)$ and fix a point $\left(v_{q}, p\right) \in T_{P} Q$ that projects onto $\left(v_{[q]_{G}},[p]_{G_{\mu}}\right)$, i.e. $T \pi^{Q}\left(v_{q}\right)=v_{[q]}$ and $p \in[p]_{G_{\mu}}$. Due to the $G$-regularity of $L$, there exists a unique element $\xi$ in $\mathfrak{g}$ such that $J_{L, \delta}\left(v_{q}+\xi_{Q}(q), p\right)=\mu$. The orbit of the point $\left(v_{q}+\xi_{Q}(q), p\right)$ under the $G_{\mu}$-action determines an element in $J_{L, \delta}^{-1}(\mu) / G_{\mu}$. We will show that this construction is independent of the chosen point $\left(v_{q}, p\right)$, and therefore defines a map $\Delta_{\mu}: T_{P / G_{\mu}}(Q / G) \rightarrow J_{L, \delta}^{-1}(\mu) / G_{\mu}$ which is the inverse to $\Pi_{\mu}$.

To show that the construction is independent of the chosen point $\left(v_{q}, p\right)$ in $T_{P} Q$, we choose any other point $\left(v_{q}^{\prime}, p^{\prime}\right)$ in $T_{P} Q$ projecting onto $\left(v_{[q]_{G}},[p]_{G_{\mu}}\right)$. The point is of the form $\left(v_{q}^{\prime}, p^{\prime}\right)=$ $\left(\Psi_{g}^{T Q}\left(v_{q}+\eta_{Q}(q)\right), p g\right)$ for some $g \in G_{\mu}$ and $\eta \in \mathfrak{g}$. We now repeat the previous construction applied to $\left(v_{q}^{\prime}, p^{\prime}\right)$ : we consider a unique element $\xi^{\prime} \in \mathfrak{g}$ for which $J_{L, \delta}\left(\Psi_{g}^{T Q}\left(v_{q}+\eta_{Q}(q)\right)+\right.$ $\left.\xi_{Q}^{\prime}(q g), p g\right)=\mu$. Due to the equivariance of $J_{L, \delta}$, we conclude that

$$
\mu=J_{L, \delta}\left(\Psi_{g}^{T Q}\left(v_{q}+\eta_{Q}(q)\right)+\xi_{Q}^{\prime}(q g), p g\right)=\operatorname{Ad}_{g}^{*} \cdot J_{L, \delta}\left(v_{q}+\left(\eta+\operatorname{Ad}_{g} \xi^{\prime}\right)_{Q}(q), p\right)-\sigma_{\delta}\left(g^{-1}\right) .
$$


Since $g \in G_{\mu}$, we may conclude that $J_{L, \delta}\left(v_{q}+\left(\eta+\operatorname{Ad}_{g} \xi^{\prime}\right)_{Q}(q), p\right)=\mu$ and therefore $\eta+\operatorname{Ad}_{g} \xi^{\prime}=\xi$. This implies that $\left(v_{q}^{\prime}+\xi_{Q}^{\prime}(q), p^{\prime}\right)=\Psi_{g}^{T_{P} Q}\left(v_{q}+\xi_{Q}(q), p\right)$ for $g \in G_{\mu}$ such that $p^{\prime}=p g$. This concludes the proof.

The previous proposition guarantees that for a given $G$-regular Lagrangian and a bundle adapted coordinate chart $\left(x^{r}, g^{m}\right)$ in $Q \rightarrow Q / G$ and $\left(x^{r}, g^{m}, p^{a}\right)$ in $P \rightarrow Q$, the functions $\left(x^{r}, \dot{x}^{r}, g^{m}, p^{a}\right)$ determine a coordinate chart in $J_{L, \delta}^{-1}(\mu)$. This is useful in the following.

Step 2: the reduced symplectomorphism. We will now introduce a new Lagrangian $\tilde{L}$ on $T_{P / G_{\mu}}(Q / G)$ with the property that its Legendre transform $\mathbb{F} \tilde{L}: T_{P / G_{\mu}}(Q / G) \rightarrow T_{P / G_{\mu}}^{*}(Q / G)$ is precisely the reduced Legendre transform $\left[(\mathbb{F} L)_{\mu}\right]$, more specifically $\mathbb{F} \tilde{L}=\left[\phi_{\mu}^{\mathcal{A}}\right] \circ\left[(\mathbb{F} L)_{\mu}\right] \circ \Delta_{\mu}$.

As is already described in [10], the structure of the Lagrangian $\tilde{L}$ is completely determined by the cotangent bundle reduction scheme, and in particular by the shift map $\phi_{\mu}^{\mathcal{A}}$ from $J_{\delta}^{-1}(\mu) \rightarrow$ $V_{P}^{0} \pi^{Q}$. To make this statement more precise, we remark that $\left[\phi_{\mu}^{\mathcal{A}}\right] \circ\left[(\mathbb{F} L)_{\mu}\right]$ is the quotient map of $\phi_{\mu}^{\mathcal{A}} \circ \mathbb{F} L$. Thus, if $\left[\left(v_{q}, p\right)\right]_{G_{\mu}}$ is arbitrary in $J_{L, \delta}^{-1}(\mu) / G_{\mu}$, then $\left[\phi_{\mu}^{\mathcal{A}}\right] \circ\left[(\mathbb{F} L)_{\mu}\right]\left(\left[\left(v_{q}, p\right)\right]_{G_{\mu}}\right)$ is the quotient of

$$
\mathbb{F} L\left(v_{q}, p\right)-\left(\left\langle\mu+\delta(p), \mathcal{A}^{Q}(q)\right\rangle, p\right) .
$$

The latter is the fibre derivative of a new function on $T_{P} Q$, namely $L\left(v_{q}, p\right)-\left\langle\mu+\delta(p), \mathcal{A}^{Q}(q)\left(v_{q}\right)\right\rangle$. This function is clearly $G_{\mu}$-invariant.

Definition 17. The function $\tilde{L}$ is defined as the pull-back under $\Delta_{\mu}$ of the quotient map of the restriction to $J_{L, \delta}^{-1}(\mu)$ of the function

$$
\left(v_{q}, p\right) \mapsto L\left(v_{q}, p\right)-\left\langle\mu+\delta(p), \mathcal{A}^{Q}(q)\left(v_{q}\right)\right\rangle .
$$

Lemma 2. $\mathbb{F} \tilde{L}=\left[\phi_{\mu}^{\mathcal{A}}\right] \circ\left[(\mathbb{F} L)_{\mu}\right] \circ \Delta_{\mu}$.

Proof. Fix elements $\left(v_{[q]_{G}},[p]_{G_{\mu}}\right) \in T_{P / G_{\mu}}(Q / G)$ and fix a representative $\left(v_{q}, p\right) \in J_{L, \delta}^{-1}(\mu)$ in the orbit $\Delta_{\mu}\left(v_{[q]_{G}},[p]_{G_{\mu}}\right) \in J_{L, \delta}^{-1}(\mu) / G_{\mu}$. By definition of the maps involved, we have

$$
\begin{aligned}
\left(\left[\phi_{\mu}^{\mathcal{A}}\right] \circ\left[(\mathbb{F} L)_{\mu}\right] \circ \Delta_{\mu}\right)\left(v_{[q]_{G}},[p]_{G_{\mu}}\right) & =\left(\pi_{\mu}^{0} \circ \phi_{\mu}^{\mathcal{A}}\right)\left(\mathbb{F} L\left(v_{q}, p\right)\right) \\
& =\pi_{\mu}^{0}\left(\mathbb{F} L\left(v_{q}, p\right)-\left(\left\langle\mu+\delta(p), \mathcal{A}^{Q}(q)\right\rangle, p\right)\right) .
\end{aligned}
$$

Next we study the fibre derivative of the map $\tilde{L}$. Fix a point $\left(w_{[q]_{G}},[p]_{G_{\mu}}\right)$ and we compute

$$
\left\langle\mathbb{F} \tilde{L}\left(v_{[q]_{G}},[p]_{G_{\mu}}\right),\left(w_{[q]_{G}},[p]_{G_{\mu}}\right)\right\rangle=\left.\frac{d}{d u}\right|_{u=0} \tilde{L}\left(v_{[q]_{G}}+u w_{[q]_{G}},[p]_{G_{\mu}}\right) .
$$

We construct a curve $u \mapsto \zeta(u)$ in $J_{L, \delta}^{-1}(\mu)$ that projects onto the curve $u \mapsto \Delta_{\mu}\left(v_{[q]_{G}}+\right.$ $\left.u w_{[q]_{G}},[p]_{G_{\mu}}\right)$ in $J_{L, \delta}^{-1}(\mu) / G_{\mu}$ such that $\zeta(0)=\left(v_{q}, p\right)$ and $\dot{\zeta}(0)$ is vertical to the projection $\rho_{2} \circ i_{\mu}: J_{L, \delta}^{-1}(\mu) \rightarrow P$ (recall that $\rho_{2}: T_{P} Q \rightarrow P$ is the projection onto the second factor in the fibred product).

The existence of such a curve is best shown in a specific coordinate chart (see above) for $J_{L}^{-1}(\mu):\left(x^{r}, \dot{x}^{r}, g^{m}, p^{a}\right)$ where the index $r=1, \ldots, \operatorname{dim} Q / G, m=1, \ldots, \operatorname{dim} G$ and $a=$ $1, \ldots, \operatorname{dim} P-\operatorname{dim} Q$. Note that $\left(x^{r}, \dot{x}^{r}\right)$ is a coordinate chart in $T(Q / G)$. In these coordinates, we let $\left(v_{q}, p\right)=\left(x_{0}^{r}, \dot{x}_{0}^{r}, g_{0}^{m}, p_{0}^{a}\right)$ and $w_{[q]_{G}}=\left(x_{0}^{r}, w_{0}^{r}\right)$. We define the curve $\zeta(u)$ to be the curve $u \mapsto\left(x_{0}^{r}, \dot{x}_{0}^{r}+u w_{0}^{i}, g_{0}^{m}, p_{0}^{a}\right)$. Then the tangent vector to $T \rho_{1}(\dot{\zeta}(0))$ is the vertical lift of some $w_{q} \in T_{q} Q$ with $T \pi^{Q}\left(w_{q}\right)=w_{[q]_{G}}$. 
Finally, from the definition of $\tilde{L}$ and the fibre derivative $\mathbb{F} \tilde{L}$ we obtain

$$
\begin{aligned}
\left\langle\mathbb{F} \tilde{L}\left(v_{[q]_{G}},[p]_{G_{\mu}}\right),\left(w_{[q]_{G}},[p]_{G_{\mu}}\right)\right\rangle & =\left.\frac{d}{d u}\right|_{u=0}\left(L-\left\langle\mu+\delta, \mathcal{A}^{Q}\right\rangle\right)(\zeta(u)) \\
& =\left\langle\mathbb{F} L\left(v_{q}, p\right)-\left(\left\langle\mu+\delta(p), \mathcal{A}^{Q}(q)\right\rangle, p\right),\left(w_{q}, p\right)\right\rangle \\
& =\left\langle\pi_{\mu}^{0}\left(\mathbb{F} L\left(v_{q}, p\right)-\left(\left\langle\mu+\delta(p), \mathcal{A}^{Q}(q)\right\rangle, p\right)\right),\left(w_{[q]_{G}},[p]_{G_{\mu}}\right)\right\rangle,
\end{aligned}
$$

since $\mathbb{F} L\left(v_{q}, p\right)-\left(\left\langle\mu+\delta(p), \mathcal{A}^{Q}(q)\right\rangle, p\right) \in V_{P}^{0} \pi^{Q}$. This concludes the proof.

Step 3: the reduced energy Hamiltonian. The third and last step concerns the specific reduced dynamics. We have to relate the energy $E_{L}$ on $T_{P} Q$ to the energy of the Routhian $\tilde{L}$ on $T_{P / G_{\mu}}(Q / G)$. In the following Lemma we again use the notations from Theorem 2 applied to the reduction of the symplectic structure on $\left(T_{P} Q, \Omega^{L, \mathcal{B}}\right)$.

Lemma 3. The energy $E_{\tilde{L}}$ is the reduced Hamiltonian, i.e. it satisfies:

$$
\left(\Pi_{\mu} \circ \pi_{\mu}\right)^{*} E_{\tilde{L}}=i_{\mu}^{*} E_{L},
$$

with $\Pi_{\mu} \circ \pi_{\mu}: J_{L, \delta}^{-1}(\mu) \rightarrow J_{L, \delta}^{-1}(\mu) / G_{\mu} \rightarrow T_{P / G_{\mu}}(Q / G)$ and $i_{\mu}: J_{L, \delta}^{-1}(\mu) \rightarrow T_{P} Q$.

Proof. Let $\left(v_{q}, p\right) \in J_{L, \delta}^{-1}(\mu)$, such that $\left(\Pi_{\mu} \circ \pi_{\mu}\right)\left(v_{q}, p\right)=\left(v_{[q]_{G}},[p]_{G_{\mu}}\right)$. Then

$$
\begin{aligned}
i_{\mu}^{*} E_{L}\left(v_{q}, p\right) & =\left\langle\mathbb{F} L\left(v_{q}, p\right),\left(v_{q}, p\right)\right\rangle-L\left(v_{q}, p\right) \\
& =\left\langle\left(\phi_{\mu}^{\mathcal{A}} \circ(\mathbb{F} L)_{\mu}\right)\left(v_{q}, p\right),\left(v_{q}, p\right)\right\rangle-\left(L\left(v_{q}, p\right)-\left\langle\mu+\delta(p), \mathcal{A}^{Q}(q)\left(v_{q}\right)\right\rangle\right) \\
& =\left\langle\left(\left[\phi_{\mu}^{\mathcal{A}}\right] \circ\left[(\mathbb{F} L)_{\mu}\right] \circ \Delta_{\mu}\right)\left(v_{[q]_{G}},[p]_{G_{\mu}}\right),\left(v_{[q]_{G}},[p]_{G_{\mu}}\right)\right\rangle-\tilde{L}\left(v_{[q]_{G}},[p]_{G_{\mu}}\right) .
\end{aligned}
$$

Using the result from Lemma 2 this concludes the proof.

Routh reduction. The previous three steps are summarized in the following theorem.

Theorem 5 (Routh reduction for magnetic Lagrangian systems). Let $(\epsilon: P \rightarrow Q, L, \mathcal{B})$ be a hyperregular, $G$-invariant and $G$-regular magnetic Lagrangian system and let $\delta$ be a $\mathcal{B} \mathfrak{g}$-potential of the magnetic term $\mathcal{B}$ with 1-cocycle $\sigma_{\delta}$.

1. Let $\mu \in \mathfrak{g}^{*}$ be a regular value of the momentum map $J_{L, \delta}$ and let $G_{\mu}$ be the isotropy subgroup of $\mu$ w.r.t. the affine action on $\mathfrak{g}^{*}$ with 1 -cocycle $-\sigma_{\delta}$, i.e. $g \in G_{\mu}$ if and only if $\mu=\operatorname{Ad}_{g}^{*} \mu-\sigma_{\delta}\left(g^{-1}\right)$.

2. Fix a connection $\mathcal{A}^{Q}$ on $\pi^{Q}: Q \rightarrow Q / G$ and let $\mathcal{A}^{P}$ be the corresponding connection on $P \rightarrow P / G$. Compute the restriction of the $G_{\mu}$-invariant function $L\left(v_{q}, p\right)-\langle\mu+$ $\left.\delta(p), \mathcal{A}^{Q}(q)\left(v_{q}\right)\right\rangle$ to $J_{L, \delta}^{-1}(\mu)$ and let $\tilde{L}$ be its quotient to $T_{P / G_{\mu}}(Q / G) \cong J_{L, \delta}^{-1}(\mu) / G_{\mu}$.

3. Compute $\tilde{\mathcal{B}}$ as the projection to $P / G_{\mu}$ of the 2 -form $\mathcal{B}+d\left\langle\mu+\delta, \mathcal{A}^{P}\right\rangle$.

4. Consider the magnetic Lagrangian system: $\left(\tilde{\epsilon}_{\mu}: P / G_{\mu} \rightarrow Q / G, \tilde{L}, \tilde{\mathcal{B}}\right)$.

This reduced magnetic Lagrangian system is hyperregular and every solution $p(t) \in P$ to the Euler-Lagrange equations for $(\epsilon, L, \mathcal{B})$ with momentum $\mu$ projects under $P \rightarrow P / G_{\mu}$ to a solution of the Euler-Lagrange equations for $\left(\tilde{\epsilon}_{\mu}: P / G_{\mu} \rightarrow Q / G, \tilde{L}, \tilde{B}\right)$. Conversely, every solution to the Euler-Lagrange equations for $\left(\tilde{\epsilon}_{\mu}: P / G_{\mu} \rightarrow Q / G, \tilde{L}, \tilde{B}\right)$ is the projection of a solution to the Euler-Lagrange equations for $(\epsilon, L, \mathcal{B})$ with momentum $\mu$.

It is possible to say more about the structure of $\tilde{\mathcal{B}}$ and its relation to the connection $\mathcal{A}^{Q}$. This and reconstruction aspects fall out of the scope of this paper. We refer to [11] where these topics are described in more detail. They carry over to this more general framework in a straightforward way. 


\subsection{Reduction of magnetic Lagrangian systems on Lie groups}

Consider a magnetic Lagrangian system on $P=Q=G$, i.e. the configuration space is a Lie group $G$. We start from a function $\ell$ on $\mathfrak{g}$ and with it we associate a Lagrangian $L$ on $T G$ by right multiplication $L\left(g, v_{g}\right)=\ell\left(v_{g} g^{-1}\right)$. By definition $L$ is invariant under the right action of $G$ on itself. We assume that a magnetic 2 -form $\mathcal{B}$ is given which is invariant under right multiplication and admits a $\mathcal{B g}$-potential $\delta: G \rightarrow \mathfrak{g}^{*}$. We first rephrase some definitions in this specific setting.

1. The 1-cocycle $\sigma_{\delta}: G \rightarrow \mathfrak{g}^{*}$ satisfies $\sigma_{\delta}(g)=\delta\left(h g^{-1}\right)-\operatorname{Ad}_{g^{-1}}^{*} \delta(h)$, for arbitrary $h \in G$. If we let $h=e$, then $\sigma_{\delta}(g)=\delta\left(g^{-1}\right)-\operatorname{Ad}_{g^{-1}}^{*} \delta(e)$ or equivalently, $\operatorname{Ad}_{g}^{*} \sigma_{\delta}(g)=\operatorname{Ad}_{g}^{*} \delta\left(g^{-1}\right)-\delta(e)$. Similarly if we let $h=g$, then $\sigma_{\delta}(g)=\delta(e)-\operatorname{Ad}_{g^{-1}}^{*} \delta(g)$. Since $\delta$ is determined up to a constant, we may assume without loss of generality that $\delta(e)=0$.

2. The associated 2-cocycle $\Sigma_{\delta}(\xi, \eta)=\xi_{G}\left(\delta_{\eta}\right)-\delta_{[\xi, \eta]}$.

3. We use the right identification of $T G$ with $G \times \mathfrak{g}$, i.e. $\left(g, v_{g}\right)$ is mapped to $\left(g, v_{g} g^{-1}\right) \in G \times \mathfrak{g}$. The right action of $G$ on $T G$ equals right multiplication in the first factor of $G \times \mathfrak{g}$ under this identification.

4. We use the Maurer-Cartan principal connection on $G \rightarrow G / G: \mathcal{A}(g)\left(v_{g}\right)=g^{-1} v_{g}$. In the right identification, the connection corresponds to the map $(g, \xi) \in G \times \mathfrak{g} \mapsto \operatorname{Ad}_{g^{-1}} \xi \in \mathfrak{g}$.

5. The momentum map $J_{L, \delta}: T G \rightarrow \mathfrak{g}^{*}$ equals $J_{L, \delta}(g, \xi g)=\operatorname{Ad}_{g}^{*} \mathbb{F} \ell(\xi)-\delta(g)$, for $(g, \xi) \in G \times \mathfrak{g}$ arbitrary. If $L$ is $G$-regular then $\mathbb{F} \ell$ is invertible, i.e. there exists a function $\chi: \mathfrak{g}^{*} \rightarrow \mathfrak{g}$ such that $\mathbb{F} \ell(\chi(\nu))=\nu$.

6. The affine action on $\mathfrak{g}^{*}$ is $(g, \mu) \mapsto \operatorname{Ad}_{g}^{*} \mu-\sigma_{\delta}\left(g^{-1}\right)$.

7. The isotropy group $G_{\mu}$ consists of group elements $g$ such that $\mu+\delta(g)=\operatorname{Ad}_{g}^{*} \mu$.

8. The quotient $G / G_{\mu}$ (right coset space) can be identified with $\tilde{\mathcal{O}}_{\mu}$, i.e. $[g]_{G_{\mu}} \in G / G_{\mu}$ is mapped onto $\nu=\operatorname{Ad}_{g^{-1}}^{*} \mu-\sigma_{\delta}(g)=\operatorname{Ad}_{g^{-1}}^{*}(\mu+\delta(g))$. A tangent vector to $G / G_{\mu}$ at $[g]_{G_{\mu}}$ which is the projection of $\xi g$ is mapped to a vector $\dot{\nu}=-\operatorname{ad}_{\xi}^{*} \nu+i_{\xi} \Sigma_{\delta}$ in $\mathfrak{g}^{*}$.

Lemma 4. The 2 -form $\mathcal{B}+d(\langle\mu+\delta, \mathcal{A}\rangle)$ reduces to the Kirillov-Kostant-Souriau symplectic 2 -form on $\tilde{\mathcal{O}}_{\mu} \cong G / G_{\mu}$.

Proof. Let $g$ be arbitrary, and let $v_{g}=\xi g, w_{g}=\eta g$ be two tangent vectors in $T_{g} G$ with $\xi, \eta \in \mathfrak{g}$ arbitrary. Note that $v_{g}=\xi_{G}^{\prime}(g)$ with $\xi^{\prime}=\operatorname{Ad}_{g^{-1}} \xi$, and similarly $w_{g}=\eta_{G}^{\prime}(g)$ with $\eta^{\prime}=\operatorname{Ad}_{g^{-1}} \eta$. Then

$$
\mathcal{B}(g)\left(v_{g}, w_{g}\right)=\mathcal{B}(g)\left(\xi_{G}^{\prime}(g), \eta_{G}^{\prime}(g)\right)=\eta_{G}^{\prime}\left(\delta_{\xi^{\prime}}\right)(g) .
$$

On the other hand

$$
d(\langle\mu+\delta, \mathcal{A}\rangle)(g)\left(v_{g}, w_{g}\right)=\xi_{G}^{\prime}\left(\left\langle\mu+\delta, \eta^{\prime}\right\rangle\right)(g)-\eta_{G}^{\prime}\left(\left\langle\mu+\delta, \xi^{\prime}\right\rangle\right)(g)-\left\langle\mu+\delta(g),\left[\xi^{\prime}, \eta^{\prime}\right]\right\rangle .
$$

Before continuing, we compute the equivariance of the 2-cocycle $\Sigma_{\delta}\left(\xi^{\prime}, \eta^{\prime}\right)$ :

$$
\begin{aligned}
\Sigma_{\delta}\left(\xi^{\prime}, \eta^{\prime}\right) & =-\frac{d}{d s}\left\langle\sigma_{\delta}\left(g^{-1} \exp s \xi g\right), \eta^{\prime}\right\rangle=-\frac{d}{d s}\left\langle\sigma_{\delta}\left(g^{-1} \exp s \xi\right)+\operatorname{Ad}_{\exp -s \xi g}^{*} \sigma_{\delta}(g), \eta^{\prime}\right\rangle \\
& =-\frac{d}{d s}\left\langle\sigma_{\delta}\left(g^{-1}\right)+\operatorname{Ad}_{g}^{*} \sigma_{\delta}(\exp s \xi)+\operatorname{Ad}_{g}^{*} \operatorname{Ad}_{\exp -s \xi}^{*} \sigma_{\delta}(g), \operatorname{Ad}_{g^{-1}} \eta\right\rangle \\
& =\Sigma_{\delta}(\xi, \eta)+\left\langle\sigma_{\delta}(g),[\xi, \eta]\right\rangle .
\end{aligned}
$$

Summarizing, we have

$$
(\mathcal{B}+d(\langle\mu+\delta, \mathcal{A}\rangle))(g)\left(v_{g}, w_{g}\right)=\xi_{G}^{\prime}\left(\left\langle\mu+\delta, \eta^{\prime}\right\rangle\right)(g)-\left\langle\mu+\delta(g),\left[\xi^{\prime}, \eta^{\prime}\right]\right\rangle
$$




$$
\begin{aligned}
& =\Sigma_{\delta}\left(\xi^{\prime}, \eta^{\prime}\right)+\delta_{\left[\xi^{\prime}, \eta^{\prime}\right]}(g)-\left\langle\mu+\delta(g),\left[\xi^{\prime}, \eta^{\prime}\right]\right\rangle \\
& =\Sigma_{\delta}(\xi, \eta)-\left\langle\operatorname{Ad}_{g^{-1}}^{*} \mu-\sigma_{\delta}(g),[\xi, \eta]\right\rangle .
\end{aligned}
$$

This 2-form is reducible to a 2 -form on $\tilde{\mathcal{O}}_{\mu}$. If we use the isomorphism $G / G_{\mu} \rightarrow \tilde{\mathcal{O}}_{\mu}$ introduced above, the 2 -form reduces to $\tilde{\mathcal{B}}(\nu)\left(\dot{\nu}, \dot{\nu}^{\prime}\right)=\langle\dot{\nu}, \eta\rangle$ with $\eta \in \mathfrak{g}$ such that $\dot{\nu}^{\prime}=-\operatorname{ad}_{\eta}^{*} \nu+i_{\eta} \Sigma_{\delta}$.

We conclude by computing the Routhian $\tilde{L}$ as a function on $\tilde{\mathcal{O}}_{\mu}$. By definition, it equals the reduction to $\tilde{\mathcal{O}}_{\mu}$ of $L-\left.\langle\mu+\delta, \mathcal{A}\rangle\right|_{J_{L, \delta}=\mu}$. In the right identification, the level set $J_{L, \delta}\left(g, v_{g}\right)=\mu$ is precisely $\operatorname{Ad}_{g^{-1}}^{*}(\mu+\delta(g))=\mathbb{F} \ell(\xi)$, with $\xi g=v_{g}$. If we set $\nu=\operatorname{Ad}_{g^{-1}}^{*}(\mu+\delta(g)) \in \tilde{\mathcal{O}}_{\mu}$, the fixed momentum condition is $\xi=\chi(\nu)$. The Routhian $\tilde{L}(\nu)$ becomes in the right identification

$$
\tilde{L}(\nu)=\ell(\chi(\nu))-\langle\nu, \chi(\nu)\rangle \text {. }
$$

By application of the chain rule, it easily follows that $\left\langle d \tilde{L}(\nu), \dot{\nu}^{\prime}\right\rangle=-\left\langle\dot{\nu}^{\prime}, \chi(\nu)\right\rangle$. The reduced Euler-Lagrange equations are

$$
i_{\dot{\nu}} \tilde{\mathcal{B}}(\nu)=d \tilde{L}(\nu) \quad \text { or } \quad \dot{\nu}=-\operatorname{ad}_{\chi(\nu)}^{*} \nu+i_{\chi(\nu)} \Sigma_{\delta} .
$$

For later purpose, we remark that for a left action and $\ell$ originating from a left invariant Lagrangian, the reduced equations are $\dot{\nu}=\operatorname{ad}_{\chi(\nu)}^{*} \nu-i_{\chi(\nu)} \Sigma_{\delta}$ (here the 1-cocycle satisfies $\sigma_{\delta}(g)=$ $\delta(g))$.

\section{Routh reduction by stages}

In reduction by stages, we study the reduction of a $G$-invariant system (symplectic or Lagrangian) under the action of the full group $G$ and under the induced action w.r.t. a normal subgroup $K \triangleleft G$. We shall adopt as far as possible the notations used in [12]. A detailed construction of the following definitions is found in this reference.

\section{Definition 18.}

1. The Lie-algebra of $K$ is $\mathfrak{K}$ and $i$ denotes the injection $i: \mathfrak{K} \rightarrow \mathfrak{g}$ with dual $i^{*}: \mathfrak{g}^{*} \rightarrow \mathfrak{K}^{*}$.

2. The group $G$ acts on $\mathfrak{K}$ by restriction of the adjoint action. The induced action of $G$ on $\mathfrak{K}^{*}$ is denoted by the same symbol $\mathrm{Ad}^{*}: G \times \mathfrak{K}^{*} \rightarrow \mathfrak{K}^{*}$.

3. $\mu$ denotes an element in $\mathfrak{g}^{*}$ and $\nu \in \mathfrak{K}^{*}$. Then $G_{\mu}$ is the isotropy subgroup of $\mu$ under the $\mathrm{Ad}^{*}$-action of $G$ on $\mathfrak{g}^{*} ; G_{\nu}$ is the isotropy subgroup of $\nu$ under the $\mathrm{Ad}^{*}$-action of $G$ on $\mathfrak{K}^{*}$ obtained as the dual of the restricted $A d$-action of $G$ on $\mathfrak{K}$; and $K_{\nu}$ is the isotropy of $\nu$ w.r.t. to standard coadjoint action of $K$ on $\mathfrak{K}^{*}$. These groups satisfy $G_{\nu} \cap K=K_{\nu}$ and $K_{\nu}$ is normal in $G_{\nu}$.

4. $\mathfrak{g}_{\nu}$ and $\mathfrak{K}_{\nu}$ denote the Lie algebras of $G_{\nu}$ and $K_{\nu}$ respectively. $\bar{G}_{\nu}$ denotes the quotient group $G_{\nu} / K_{\nu}$ and its Lie algebra equals $\overline{\mathfrak{g}}_{\nu}=\mathfrak{g}_{\nu} / \mathfrak{K}_{\nu}$.

5. The projections onto the quotient groups are denoted by $r: G \rightarrow \bar{G}=G / K$ and $r_{\nu}: G_{\nu} \rightarrow$ $\bar{G}_{\nu}$, and on the level of the Lie algebra: $r^{\prime}: \mathfrak{g} \rightarrow \overline{\mathfrak{g}}=\mathfrak{g} / \mathfrak{K}$ and $r_{\nu}^{\prime}: \mathfrak{g}_{\nu} \rightarrow \overline{\mathfrak{g}}_{\nu}$. The inclusion map $G_{\nu} \rightarrow G$ induces a map $k_{\nu}: \mathfrak{g}_{\nu} \rightarrow \mathfrak{g}$, with its dual $k_{\nu}^{*}: \mathfrak{g}^{*} \rightarrow \mathfrak{g}_{\nu}^{*}$.

6. $\rho$ denotes an element in $\overline{\mathfrak{g}}_{\nu}^{*}$.

In [12] symplectic reduction by stages is performed under the condition of a so-called 'stages hypothesis'. An element $\mu \in \mathfrak{g}^{*}$ is said to satisfy the stages hypothesis if for any $\mu^{\prime} \in \mathfrak{g}^{*}$ satisfying $\left.\mu\right|_{\mathfrak{K}}=\left.\mu^{\prime}\right|_{\mathfrak{K}}=\nu$ and $\left.\mu\right|_{\mathfrak{g}_{\nu}}=\left.\mu^{\prime}\right|_{\mathfrak{g}_{\nu}}=\bar{\nu}$, there exists an element $k \in K_{\nu}$ and $g \in\left(G_{\nu}\right)_{\bar{\nu}}$ such that $\operatorname{Ad}_{k g}^{*} \mu^{\prime}=\mu$. The stages hypothesis is a condition on a chosen momentum value and 
depends only on the symmetry group $G$. It was already clear in [12] that the hypothesis is automatically satisfied if $G$ is a central extension or if $G$ is a semi-direct product group. In the recent contribution [16] it has been pointed out that the hypothesis is in fact always satisfied, and that it can be taken out of the reduction by stages statements altogether. Taking advantage of this result, in this paper, we will not make further reference to the stages hypothesis.

\subsection{Symplectic reduction by stages}

Theorem 6 (Symplectic reduction by stages [12]). Let $(M, \omega)$ be a symplectic manifold with a canonical $G$-action $\Psi^{M}$ with an equivariant momentum map $J_{G}$.

1. Fix a regular value $\mu \in \mathfrak{g}^{*}$ of the momentum map and perform symplectic reduction to obtain the symplectic manifold $\left(M_{\mu}, \omega_{\mu}\right)$.

2. The restriction of the action $\Psi^{M}$ to $K$ is canonical and the map $J_{K}=i^{*} \circ J_{G}: M \rightarrow \mathfrak{K}^{*}$ determines an equivariant momentum map for this induced action. Fix a regular value $\nu$ of $J_{K}$ and perform symplectic reduction to obtain the symplectic manifold $\left(M_{\nu}, \omega_{\nu}\right)$.

3. The level set $J_{K}^{-1}(\nu)$ is $G_{\nu}$-invariant.

4. The group $\bar{G}_{\nu}=G_{\nu} / K_{\nu}$ acts on $M_{\nu}$ by projecting the restricted action of $G_{\nu}$ on $J_{K}^{-1}(\nu)$. This induced action $\Psi^{M_{\nu}}$ is free, proper and canonical on $\left(M_{\nu}, \omega_{\nu}\right)$. Assume that $K_{\nu}$ is connected.

5. Fix an element $\bar{\nu}$ in $\mathfrak{g}_{\nu}^{*}$ such that the restriction of $\left.\bar{\nu}\right|_{\mathfrak{K}_{\nu}}$ equals $\left.\nu\right|_{\mathfrak{K}_{\nu}}$. There is a well-defined momentum map $J_{\bar{G}_{\nu}}: M_{\nu} \rightarrow \overline{\mathfrak{g}}_{\nu}^{*}$ for the induced action $\Psi^{M_{\nu}}$. This momentum map is determined from $J_{G}$ and $\bar{\nu}$ and has a non-equivariance cocycle: $J_{\bar{G}_{\nu}}$ satisfies

$$
\left(r_{\nu}^{\prime}\right)^{*} \circ J_{\bar{G}_{\nu}} \circ \pi_{\nu}=k_{\nu}^{*} \circ J_{G} \circ i_{\nu}-\bar{\nu} .
$$

6. Let $\rho \in \overline{\mathfrak{g}}_{\nu}^{*}$ be a regular value for the momentum map $J_{\bar{G}_{\nu}}$ and let $\left(\bar{G}_{\nu}\right)_{\rho}$ be the isotropy subgroup of $\rho$ w.r.t. the affine action of $\bar{G}_{\nu}$ on $\overline{\mathfrak{g}}_{\nu}^{*}$. Perform symplectic reduction to obtain the symplectic manifold $\left(\left(M_{\nu}\right)_{\rho},\left(\omega_{\nu}\right)_{\rho}\right)$ with $\left(M_{\nu}\right)_{\rho}=J_{\bar{G}_{\nu}}^{-1}(\rho) /\left(\bar{G}_{\nu}\right)_{\rho}$.

If $\rho$ is chosen such that $\left(r_{\nu}^{\prime}\right)^{*} \rho=\left.\mu\right|_{\mathfrak{g}_{\nu}}-\bar{\nu}$, then there exists a symplectic diffeomorphism

$$
F:\left(M_{\mu}, \omega_{\mu}\right) \rightarrow\left(\left(M_{\nu}\right)_{\rho},\left(\omega_{\nu}\right)_{\rho}\right) .
$$

For our purpose it is also important to understand the reduction of a $G$-invariant Hamiltonian $h$ on $M$. We assume that all conditions in Theorem 6 are satisfied. First note that, by definition of the momentum maps, we have an inclusion $j_{\mu}$ of $J_{G}^{-1}(\mu)$ in $J_{K}^{-1}(\nu)$. Recall that we use $\pi_{\nu}$ for the projection $J_{K}^{-1}(\nu) \rightarrow M_{\nu}$. It was shown in [12] that the image of $\pi_{\nu} \circ j_{\mu}: J_{G}^{-1}(\mu) \rightarrow M_{\nu}$ is contained in $J_{\bar{G}_{\nu}}^{-1}(\rho)$. Moreover, this map is equivariant w.r.t. the action of $G_{\mu}$ on $J_{G}^{-1}(\mu)$ and $\left(\bar{G}_{\nu}\right)_{\rho}$ on $J_{\bar{G}_{\nu}}^{-1}(\rho)$ (this makes sense, since $G_{\mu}$ projects to a subset of $\left.\left(\bar{G}_{\nu}\right)_{\rho}\right)$. The quotient of $\pi_{\nu} \circ j_{\mu}$ is the symplectic diffeomorphism $F$ mentioned in the previous theorem (see also Fig. 4).

Let $h$ be a $G$-invariant Hamiltonian on $M$ and let $h_{\mu}$ be the function on $M_{\mu}$ obtained from $\pi_{\mu}^{*} h_{\mu}=i_{\mu}^{*} H$. On the other hand we let $h_{\nu}$ be the function satisfying $\pi_{\nu}^{*} h_{\nu}=i_{\nu}^{*} h$. This function is $\bar{G}_{\nu}$-invariant: $h_{\nu}\left([m]_{K_{\nu}}[g]_{K_{\nu}}\right)=h\left(i_{\nu}(m g)\right)=h\left(i_{\nu}(m) g\right)$, with $m \in J_{K}^{-1}(\nu)$ and $g \in G_{\nu}$ arbitrary. Note that $\left(\pi_{\nu} \circ j_{\mu}\right)^{*} h_{\nu}=i_{\mu}^{*} h$.

The Hamiltonian $h_{\nu}$ is a $\bar{G}_{\nu}$-invariant function on $\left(M_{\nu}, \omega_{\nu}\right)$. Applying the second symplectic reduction to this manifold, we obtain a new reduced Hamiltonian $\left(h_{\nu}\right)_{\rho}$ on $\left(M_{\nu}\right)_{\rho}$.

Proposition 8. $F^{*}\left(\left(h_{\nu}\right)_{\rho}\right)=h_{\mu}$. 




Figure 4. Commuting diagram relating the different reduced symplectic manifolds.

Proof. We rely on the commuting diagram in Fig. 4:

$$
\pi_{\mu}^{*}\left(F^{*}\left(\left(h_{\nu}\right)_{\rho}\right)\right)=\left(\pi_{\nu} \circ j_{\mu}\right)^{*} h_{\nu}=i_{\mu}^{*}(h) .
$$

This uniquely characterizes $F^{*}\left(\left(h_{\nu}\right)_{\rho}\right)$ as the function $h_{\mu}$.

\subsection{Routh reduction by stages}

Routh reduction by stages is symplectic reduction by stages applied to the symplectic structure of the initial Lagrangian system. In this section we show that the symplectic structures and energy hamiltonians in the different stages can in fact be associated to specific magnetic Lagrangians systems, and eventually gives us Routh reduction by stages. The symplectic reduction by stages then provides us a diffeomorphism relating the solutions of the different Euler-Lagrange equations for the Lagrangian systems in the final stages.

We start with a hyperregular Lagrangian $L$, invariant under the action of a Lie group $G$. We assume that this Lagrangian satisfies a regularity condition which is more stringent than mere $G$-regularity.

Definition 19. The Lagrangian $L$ is said to be $G$-hyperregular if for any $v_{q} \in T Q$ and any subspace $\mathfrak{K}^{\prime}<\mathfrak{g}$ with injection $i^{\prime}: \mathfrak{K}^{\prime} \rightarrow \mathfrak{g}$, the mapping $\left.i^{\prime *} \circ J_{L}\right|_{v_{q}} \circ i^{\prime}: \mathfrak{K}^{\prime} \rightarrow \mathfrak{K}^{*}$ defined by $\xi \mapsto i^{\prime *}\left(J_{L}\left(v_{q}+\left(i^{\prime}(\xi)\right)_{Q}(q)\right)\right)$ is invertible.

Lagrangians of mechanical type are $G$-hyperregular. Let $K$ be a normal subgroup of $G, \mathfrak{K}$ the Lie algebra of $K$ and $i: \mathfrak{K} \rightarrow \mathfrak{g}$ the canonical injection. Due to the hyperregularity the invariant Lagrangian $L$ is both $G$ - and $K$-hyperregular, and both $G$ - and $K$-invariant. By definition of $J_{L}$, the map $i^{*} \circ J_{L}=i^{*} \circ\left(\psi^{T Q}\right)^{*} \circ \mathbb{F} L$ is the momentum map for the $K$-action.

Theorem 7 (Routh reduction by stages). Assume $(Q, L)$ is a hyperregular, G-hyperregular and $G$-invariant Lagrangian system. Let $K$ denote a normal subgroup of $G$.

1. Let $\mu \in \mathfrak{g}^{*}$ be a regular value of the momentum map $J_{L}$ and $\mathcal{A}^{0}$ a $G$-connection on $Q$. Let $\left(Q / G_{\mu} \rightarrow Q / G, L_{0}, \mathcal{B}_{0}\right)$ be the magnetic Lagrangian system obtained by performing Routh reduction with respect to $G$.

2. Fix a regular value $\nu \in \mathfrak{K}^{*}$ of the momentum map $i^{*} \circ J_{L}$ for the $K$-action and a $K$ connection $\mathcal{A}^{1}$ on $Q$. Assume that $\mathcal{A}^{1}$ is $G$-equivariant w.r.t. the action of $G$ on $Q$ and $\mathfrak{K}^{*}$. Consider the magnetic Lagrangian system $\left(Q / K_{\nu} \rightarrow Q / K, L_{1}, \mathcal{B}_{1}\right)$ obtained by performing Routh reduction with respect to $K$.

3. $\bar{G}_{\nu}$ acts on $Q / K_{\nu}$ and $Q / K$ by projecting the induced action of $G_{\nu}$ on $Q$. These induced actions are free and proper.

4. Assume that $K_{\nu}$ is connected. Fix an element $\bar{\nu} \in \mathfrak{g}_{\nu}^{*}$ such that $\left.\bar{\nu}\right|_{\mathfrak{K}_{\nu}}=\left.\nu\right|_{\mathfrak{K}_{\nu}}$. Then the magnetic Lagrangian system $\left(Q / K_{\nu} \rightarrow Q / K, L_{1}, \mathcal{B}_{1}\right)$ is $\bar{G}_{\nu}$-invariant, $\bar{G}_{\nu}$-regular and 
admits a $\mathcal{B}_{1} \overline{\mathfrak{g}}_{\nu}$-potential $\delta_{1}$ entirely determined by the choice of $\bar{\nu}$. The potential satisfies, for arbitrary $q \in Q$

$$
\left(r_{\nu}^{\prime}\right)^{*}\left(\delta_{1}\left([q]_{K_{\nu}}\right)\right)=-\left(\psi^{Q} \circ k_{\nu}\right)^{*}\left(\left\langle\nu, \mathcal{A}^{1}(q)\right\rangle\right)+\bar{\nu}
$$

Let $J_{1}$ denote the momentum map associated with $\delta_{1}$.

5. Fix a regular value $\rho \in \overline{\mathfrak{g}}_{\nu}^{*}$ for the momentum map $J_{1}$ and let $\left(\bar{G}_{\nu}\right)_{\rho}$ be the isotropy subgroup of $\rho$ w.r.t. the affine action of $\bar{G}_{\nu}$ on $\overline{\mathfrak{g}}_{\nu}$. Fix a $\bar{G}_{\nu}$-connection on $Q / K$. Consider the magnetic Lagrangian system $\left.\left(\left(Q / K_{\nu}\right) /\left(\bar{G}_{\nu}\right)_{\rho} \rightarrow(Q / K) / \bar{G}_{\nu}\right), L_{2}, \mathcal{B}_{2}\right)$ obtained by performing Routh reduction with respected to $\bar{G}_{\nu}$.

If $\rho$ is chosen such that $\left(r_{\nu}^{\prime}\right)^{*} \rho=\left.\mu\right|_{\mathfrak{g}_{\nu}}-\bar{\nu}$, then every solution $\gamma(t) \in Q / G_{\mu}$ to the EulerLagrange equations for $\left(Q / G_{\mu} \rightarrow Q / G, L_{0}, \mathcal{B}_{0}\right)$ is mapped to a solution in $\left(Q / K_{\nu}\right) /\left(\bar{G}_{\nu}\right)_{\rho}$ to the Euler-Lagrange equations for $\left(\left(Q / K_{\nu}\right) /\left(\bar{G}_{\nu}\right)_{\rho} \rightarrow(Q / K) / \bar{G}_{\nu}, L_{2}, \mathcal{B}_{2}\right)$. Conversely, a solution in $\left(Q / K_{\nu}\right) /\left(\bar{G}_{\nu}\right)_{\rho}$ to the Euler-Lagrange equations for $\left(\left(Q / K_{\nu}\right) /\left(\bar{G}_{\nu}\right)_{\rho} \rightarrow(Q / K) / \bar{G}_{\nu}, L_{2}, \mathcal{B}_{2}\right)$ is the projection of a solution in $Q / G_{\mu}$ to the Euler-Lagrange equations for $\left(Q / G_{\mu} \rightarrow Q / G, L_{0}, \mathcal{B}_{0}\right)$.

Proof. 1 and 2 are obtained by applying Routh reduction. 3 follows from [12, p. 152]: we know that the quotient groups $\bar{G}_{\nu}=G_{\nu} / K_{\nu}$ acts in a free and proper way on the quotient space $Q / K_{\nu}$. The group $\bar{G}_{\nu}$ is a subgroup of $\bar{G}$ and acts freely and properly and $Q / K$. We now show 4 .

$\bar{G}_{\nu}$-Invariance of the Routh reduced system $\left(Q / K_{\nu} \rightarrow Q / K, L_{1}, \mathcal{B}_{1}\right)$.

Lemma 5. If the connection $\mathcal{A}^{1}$ is chosen such that it is equivariant w.r.t. the action of the full group $G$, i.e. if

$$
\left(\Psi_{g}^{Q}\right)^{*} \mathcal{A}^{1}=\operatorname{Ad}_{g^{-1}} \mathcal{A}^{1}
$$

then the magnetic Lagrangian system $\left(Q / K_{\nu} \rightarrow Q / K, L_{1}, \mathcal{B}_{1}\right)$ is $\bar{G}_{\nu^{-}}$-invariant and $\delta_{1}$ is a $\mathcal{B}_{1} \overline{\mathfrak{g}}_{\nu^{-}}$ potential.

Proof. We first show that $L_{1}$ is $\bar{G}_{\nu}$-invariant. For that purpose, we choose an arbitrary $\bar{g} \in \bar{G}_{\nu}$ and let $g \in G_{\nu}$ be a representative. Similar we choose a point $\left(v_{[q]_{K}},[q]_{K_{\nu}}\right) \in T_{Q / K_{\nu}}(Q / K)$ such that it is the projection of $v_{q} \in\left(i \circ J_{L}\right)^{-1}(\nu) \subset T Q$. By definition of the quotient action on $T_{Q / K_{\nu}}(Q / K)$, the action of $\bar{g}$ on an element $\left(v_{[q]_{K}},[q]_{K_{\nu}}\right)$ equals the projection of $v_{q} g$. We now check the invariance of $L_{1}$ at an arbitrary point in $T_{Q / K_{\nu}}(Q / K)$ :

$$
\begin{aligned}
L_{1}\left(\Psi_{\bar{g}}^{T_{Q / K_{\nu}}(Q / K)}\left(v_{[q]_{K}},[q]_{K_{\nu}}\right)\right) & =L\left(\Psi_{g}^{T Q}\left(v_{q}\right)\right)-\left\langle\nu, \mathcal{A}^{1}(q g)\left(T \Psi_{g}^{Q}\left(v_{q}\right)\right)\right\rangle \\
& =L\left(v_{q}\right)-\left\langle\operatorname{Ad}_{g^{-1}}^{*} \nu, \mathcal{A}^{1}(q)\left(v_{q}\right)\right\rangle=L_{1}\left(v_{[q]_{K}},[q]_{K_{\nu}}\right) .
\end{aligned}
$$

Next, we check the $\bar{G}_{\nu}$-invariance of $\mathcal{B}_{1}$. Recall that $\mathcal{B}_{1}$ is the projection to $Q / K_{\nu}$ of the 2 -form $d\left\langle\nu, \mathcal{A}^{1}\right\rangle$ on $Q$. We first consider the equivariance of this 2 -form under $G_{\nu}$. Let $g \in G_{\nu}$ be arbitrary, then

$$
\left(\Psi_{g}^{Q}\right)^{*}\left(d\left\langle\nu, \mathcal{A}^{1}\right\rangle\right)=d\left\langle\operatorname{Ad}_{g^{-1}}^{*} \nu, \mathcal{A}^{1}\right\rangle=d\left\langle\nu, \mathcal{A}^{1}\right\rangle .
$$

We thus obtain $G_{\nu^{-}}$-invariance for $d\left\langle\nu, \mathcal{A}^{1}\right\rangle$, and we may conclude that $\left(\Psi_{\bar{g}}^{Q / K_{\nu}}\right)^{*} \mathcal{B}_{1}=\mathcal{B}_{1}$ holds on $Q / K_{\nu}$.

The third and final step is the definition of the $\mathcal{B}_{1} \overline{\mathfrak{g}}_{\nu}$-potential. We consider an element $\bar{\xi}=[\xi]_{\mathfrak{K}_{\nu}} \in \overline{\mathfrak{g}}_{\nu}=\mathfrak{g}_{\nu} / \mathfrak{K}_{\nu}$ and let $\xi \in \mathfrak{g}_{\nu}$ be a representative. Then, by definition of $\mathcal{B}_{1}$, the 1 -form $i_{\bar{\xi}_{Q / K_{\nu}}} \mathcal{B}_{1}$ is the projection to $Q / K_{\nu}$ of the 1 -form $i_{\xi_{Q}} d\left(\left\langle\nu, \mathcal{A}^{1}\right\rangle\right)$ on $Q$ (i.e. $\xi_{Q}$ projects to $\bar{\xi}_{Q / K_{\nu}}$ ). Again we concentrate on the 1-form on $Q$ :

$$
i_{\xi_{Q}} d\left\langle\nu, \mathcal{A}^{1}\right\rangle=\mathcal{L}_{\xi_{Q}}\left(\left\langle\nu, \mathcal{A}^{1}\right\rangle\right)-d\left(i_{\xi_{Q}}\left\langle\nu, \mathcal{A}^{1}\right\rangle\right) .
$$


Since $\left\langle\nu, \mathcal{A}^{1}\right\rangle$ is $G_{\nu}$-invariant, we conclude that $i_{\xi_{Q}} d\left\langle\nu, \mathcal{A}^{1}\right\rangle=-d\left(i_{\xi_{Q}}\left\langle\nu, \mathcal{A}^{1}\right\rangle\right)$. The exact 1-from on the right gives a strong hint of the structure of the $\overline{\mathfrak{g}}_{\nu}$-potential. Assume now that we fixed an element $\bar{\nu} \in \mathfrak{g}_{\nu}^{*}$ such that $\left.\bar{\nu}\right|_{\mathfrak{K}_{\nu}}=\left.\nu\right|_{\mathfrak{K}_{\nu}}$.

The function $\delta$ on $Q$, defined by

$$
-\delta_{\xi}(q)=\left\langle\nu, \mathcal{A}^{1}(q)\left(\xi_{Q}(q)\right)\right\rangle-\langle\bar{\nu}, \xi\rangle
$$

is our candidate for the $\mathcal{B}_{1} \overline{\mathfrak{g}}_{\nu}$-potential. This statement makes sense provided that $\delta_{\xi}$ projects to a function on $Q / K_{\nu}$ and that it only depends on the equivalence class $\bar{\xi}=\xi+\mathfrak{K}_{\nu}$ of $\xi \in \mathfrak{g}_{\nu}$. The latter is a straightforward consequence of the fact that $\mathcal{A}^{1}$ is a principal $K$-connection. The $K_{\nu}$-invariance is more involved, and we rely on a result in [12]. For any $k$ in $K_{\nu}$, we have

$$
-\delta_{\xi}(q k)=\left\langle\nu, \mathcal{A}^{1}(q k)\left(\xi_{Q}(q k)\right)\right\rangle-\langle\bar{\nu}, \xi\rangle=\left\langle\nu, \mathcal{A}^{1}(q)\left(\left(\operatorname{Ad}_{k} \xi\right)_{Q}(q)\right)\right\rangle-\langle\bar{\nu}, \xi\rangle .
$$

Therefore $\delta_{\xi}$ is constant on the orbits of $K_{\nu}$ in $Q$ if $\left\langle\nu, \mathcal{A}^{1}(q)\left(\left(\xi-\operatorname{Ad}_{k} \xi\right)_{Q}(q)\right)\right\rangle$ vanishes for all $k$. To show this we introduce a function $f$ on $K_{\nu}$ given by $f(k)=\left\langle\nu, \mathcal{A}^{1}(q)\left(\left(\xi-\operatorname{Ad}_{k} \xi\right)_{Q}(q)\right)\right\rangle$ and we use similar arguments as in [12, p. 156]. If we can show that $f(e)=0,\left.d f\right|_{e}=0$ and $f\left(k_{1} k_{2}\right)=f\left(k_{1}\right)+f\left(k_{2}\right)$ for arbitrary $k_{1,2} \in K_{\nu}$, we may conclude that $f=0$ (since $K_{\nu}$ is assumed connected).

The first condition $f(e)=0$ is trivial. To check the second condition: let $\kappa \in \mathfrak{K}_{\nu}$ be arbitrary, then

$$
\left.\left.d f\right|_{e}(\kappa)=\left\langle\nu, \mathcal{A}^{1}(q)\left(-\operatorname{ad}_{\kappa} \xi\right)_{Q}(q)\right)\right\rangle=-\left\langle\nu, \operatorname{ad}_{\kappa} \xi\right\rangle=-\left\langle\operatorname{ad}_{\kappa}^{*}, \xi\right\rangle=0 .
$$

Above, we have used the fact that $K_{\nu}$ is normal in $G_{\nu}$ and that, as a consequence, the Lie bracket $[\kappa, \xi]$ is in $\mathfrak{K}_{\nu}$. Therefore the contraction of the corresponding fundamental vector field with $\mathcal{A}^{1}$ is precisely $[\kappa, \xi]$. Next, we check the third condition and compute $f\left(k_{1} k_{2}\right)$. Given the identity

$$
\xi-\operatorname{Ad}_{k_{1}} \operatorname{Ad}_{k_{2}} \xi=\xi-\operatorname{Ad}_{k_{1}} \xi+\operatorname{Ad}_{k_{1}}\left(\xi-\operatorname{Ad}_{k_{2}} \xi\right)
$$

and the fact that $k_{1} \in K_{\nu}$,

$$
\begin{aligned}
f\left(k_{1} k_{2}\right) & =\left\langle\nu, \mathcal{A}^{1}(q)\left(\left(\xi-\operatorname{Ad}_{k_{1} k_{2}} \xi\right)_{Q}(q)\right)\right\rangle \\
& =\left\langle\nu, \mathcal{A}^{1}(q)\left(\left(\xi-\operatorname{Ad}_{k_{1}} \xi\right)_{Q}(q)\right)\right\rangle+\left\langle\nu, \mathcal{A}^{1}(q)\left(\operatorname{Ad}_{k_{1}}\left(\xi-\operatorname{Ad}_{k_{2}} \xi\right)_{Q}(q)\right)\right\rangle \\
& =f\left(k_{1}\right)+\left\langle\operatorname{Ad}_{k_{1}}^{*} \nu, \mathcal{A}^{1}(q)\left(\left(\xi-\operatorname{Ad}_{k_{2}} \xi\right)_{Q}(q)\right)\right\rangle=f\left(k_{1}\right)+f\left(k_{2}\right) .
\end{aligned}
$$

This completes the proof: the $\mathfrak{g}_{\nu}^{*}$-valued function $\delta$ is shown to be projectable to a $\overline{\mathfrak{g}}_{\nu}^{*}$-valued function on $Q / K_{\nu}$. This is the sought-after potential $\delta_{1}$ : for arbitrary $q \in Q$, we have

$$
\left(r_{\nu}^{\prime}\right)^{*}\left(\delta_{1}\left([q]_{K_{\nu}}\right)\right)=-\left(\psi^{Q} \circ k_{\nu}\right)^{*}\left(\left\langle\nu, \mathcal{A}^{1}(q)\right\rangle\right)+\bar{\nu} .
$$

Symplectic structure of $\left(Q / K_{\nu} \rightarrow Q / K, L_{1}, \mathcal{B}_{1}\right)$ and symplectic reduction by stages.

Lemma 6. Apply symplectic reduction by stages to the symplectic structure associated to the $G$-invariant Lagrangian system $(Q, L)$. Identify the symplectically reduced manifold $M_{\nu}$ with the symplectic structure on $T_{Q / K_{\nu}}(Q / K)$ induced by the magnetic Lagrangian system $\left(Q / K_{\nu} \rightarrow\right.$ $\left.Q / K, L_{1}, \mathcal{B}_{1}\right)$. Then:

1. The action $\Psi^{T_{Q / K_{\nu}}(Q / K)}$ of $\bar{G}_{\nu}$ on $T_{Q / K_{\nu}}(Q / K)$ is precisely the induced action on the first reduced space $M_{\nu}$ in symplectic reduction by stages.

2. For a chosen $\bar{\nu} \in \mathfrak{g}_{\nu}^{*}$, the momentum map $J_{1}: T_{Q / K_{\nu}}(Q / K) \rightarrow \overline{\mathfrak{g}}_{\nu}^{*}$ associated with the magnetic Lagrangian system $\left(Q / K_{\nu} \rightarrow Q / K, L_{1}, \mathcal{B}_{1}\right)$ corresponds to the induced momentum map $J_{\bar{G}_{\nu}}$ from symplectic reduction by stages. 
Proof. 1. The momentum map for the $K$ action is precisely $J_{K}:=i_{\mathfrak{K}}^{*} \circ J_{L}$, with $J_{L}: T Q \rightarrow \mathfrak{g}^{*}$. By definition, the induced action of $\bar{G}_{\nu}$ on $J_{K}^{-1}(\nu) / K_{\nu}$ is obtained by projecting the action of $G_{\nu}$ on $J_{K}^{-1}(\nu)$. If we take into account that we realize the quotient manifold $J_{K}^{-1}(\nu) / K_{\nu}$ as $T_{Q / K_{\nu}}(Q / K)$, the induced action on $T_{Q / K_{\nu}}(Q / K)$ is obtained by projection of the action of $G_{\nu}$ on $T Q$ under the projection $T Q \rightarrow T_{Q / K_{\nu}}(Q / K)$. This is precisely the action we have introduced above.

2. The induced momentum map $J_{\bar{G}_{\nu}}$ is defined in the following way (we consider it directly as a function on $T_{Q / K_{\nu}}(Q / K)$ instead of on $\left.M_{\nu}\right)$ :

$$
\left\langle J_{\bar{G}_{\nu}}\left(v_{[q]_{K}},[q]_{K_{\nu}}\right), \bar{\xi}\right\rangle=\left\langle J_{L}\left(v_{q}\right), k_{\nu}(\xi)\right\rangle-\langle\bar{\nu}, \xi\rangle=\left\langle\mathbb{F} L\left(v_{q}\right), \xi_{Q}\right\rangle-\langle\bar{\nu}, \xi\rangle
$$

where $\xi \in \mathfrak{g}_{\nu}$ is arbitrary and projects to $\bar{\xi} \in \overline{\mathfrak{g}}_{\nu}, v_{q}$ projects to $\left(v_{[q]_{K}},[q]_{K_{\nu}}\right)$. By definition of the momentum map of the magnetic Lagrangian system $\left(Q / K_{\nu} \rightarrow Q / K, L_{1}, \mathcal{B}_{1}\right)$, we have

$$
\left\langle J_{1}\left(v_{[q]_{K}},[q]_{K_{\nu}}\right), \bar{\xi}\right\rangle=\left\langle\mathbb{F} L_{1}\left(v_{[q]_{K}},[q]_{K_{\nu}}\right),\left((\bar{\xi})_{Q / K},[p]_{K_{\nu}}\right)\right\rangle-\left(\delta_{1}\right)_{\bar{\xi}}\left([p]_{K_{\nu}}\right) .
$$

We now show that the right-hand side of (5) equals the right-hand side of (4). We therefore use the definition of $\mathbb{F} L_{1}$ and $\delta_{1}$ as being the projection of maps upstairs:

$$
\begin{aligned}
& \left\langle\mathbb{F} L_{1}\left(v_{[q]_{K}},[q]_{K_{\nu}}\right),\left((\bar{\xi})_{Q / K},[q]_{K_{\nu}}\right)\right\rangle=\left\langle\mathbb{F} L\left(v_{q}\right), \xi_{Q}(q)\right\rangle-\left\langle\nu, \mathcal{A}^{1}(q)\left(\xi_{Q}(q)\right)\right\rangle, \\
& \left(\delta_{1}\right)_{\bar{\xi}}\left([q]_{K_{\nu}}\right)=\delta_{\xi}(p)=-\left\langle\nu, \mathcal{A}^{1}(q)\left(\xi_{Q}(q)\right)\right\rangle+\langle\bar{\nu}, \xi\rangle .
\end{aligned}
$$

Finally, before we can reapply Routh reduction for the second stage, we need to check that $L_{1}$ is $\bar{G}_{\nu}$-regular.

Lemma 7. The magnetic Lagrangian system $\left(Q / K_{\nu} \rightarrow Q / K, L_{1}, \mathcal{B}_{1}\right)$ is $\bar{G}_{\nu}$-regular.

Proof. We have to show that, for any $\left(v_{[q]_{K}},[q]_{K_{\nu}}\right)$ the map

$$
\overline{\mathfrak{g}}_{\nu} \rightarrow \overline{\mathfrak{g}}_{\nu}^{*} ; \quad \bar{\xi} \mapsto J_{1}\left(v_{[q]_{K}}+\bar{\xi}_{Q / K}\left([q]_{K}\right),[q]_{K_{\nu}}\right)
$$

is invertible. Let $v_{q}$ determine a tangent vector in $J_{K}^{-1}(\nu)$ representing $\left(v_{[q]_{K}},[q]_{K_{\nu}}\right)$. Let $\eta$ denote an arbitrary element in $\overline{\mathfrak{g}}_{\nu}^{*}$. Due to the assumed $G$-hyperregularity, there is a unique $\xi \in \mathfrak{g}_{\nu}$ such that $k_{\nu}^{*}\left(J_{L}\left(v_{q}+\xi_{Q}(q)\right)\right)=\left(r_{\nu}^{\prime}\right)^{*} \eta+\bar{\nu}$. The projection $\bar{\xi}=r_{\nu}^{\prime}(\xi)$ of $\xi$ defines the inverse element for $\eta$, since it is such that

$$
k_{\nu}^{*}\left(J_{L}\left(v_{q}+\xi_{Q}(q)\right)\right)-\bar{\nu}=\left(r_{\nu}^{\prime}\right)^{*} J_{1}\left(v_{[q]_{K}}+\bar{\xi}_{Q / K}\right)=\left(r_{\nu}^{\prime}\right)^{*} \eta
$$

Symplectic and Routh reduction by stages. Summarizing the above lemmas, we conclude that the magnetic Lagrangian system $\left(Q / K_{\nu} \rightarrow Q / K, L_{1}, \mathcal{B}_{1}\right)$ is amenable to Routh reduction and that the symplectic structure and momentum map associated to this Lagrangian system correspond to the symplectic structure and momentum map encountered in symplectic reduction by stages. If $\rho$ is chosen such that the compatibility relation $\left(r_{\nu}^{\prime}\right)^{*} \rho=\left.\mu\right|_{\mathfrak{g}_{\nu}}-\bar{\nu}$ holds, then from symplectic reduction by stages we have that the symplectic structures associated to $\left(Q / G_{\mu} \rightarrow Q / G, L_{0}, \mathcal{B}_{0}\right)$ and $\left(\left(Q / K_{\nu}\right) /\left(\bar{G}_{\nu}\right)_{\rho} \rightarrow(Q / K) / \bar{G}_{\nu}, L_{2}, \mathcal{B}_{2}\right)$ are symplectically diffeomorphic by means of the symplectic diffeomorphism $F$ introduced earlier. From Proposition 8 it follows that $F^{*} E_{L_{2}}=E_{L_{0}}$ and therefore the corresponding Hamiltonian vector fields are $F$-related. We define a map $\tau: Q / G_{\mu} \rightarrow\left(Q / K_{\nu}\right) /\left(\bar{G}_{\nu}\right)_{\rho}$ as $\tau\left([q]_{G_{\mu}}\right)=\left[[q]_{K_{\nu}}\right]_{\left(\bar{G}_{\nu}\right)_{\rho}}$. The map is well-defined since $G_{\mu}$ is a subgroup of $G_{\nu}$ and since $r_{\nu}\left(G_{\mu}\right) \subset\left(\bar{G}_{\nu}\right)_{\rho}$.

Lemma 8. The symplectic diffeomorphism $F$ is fibred over $\tau$. 


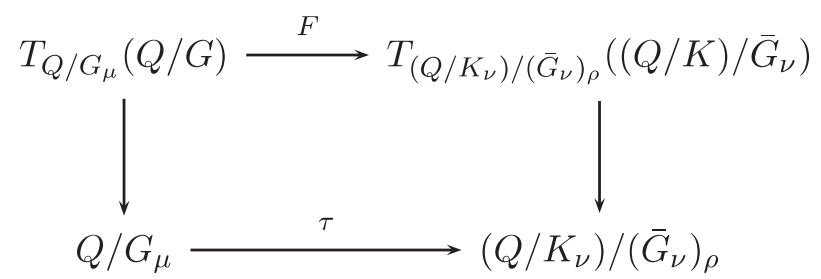

Figure 5. Fibration of the symplectic diffeomorphism $F$.

Proof. We recall the definition of the map $F$ : fix an element $\left(v_{[q]_{G}},[q]_{G_{\mu}}\right)$ and let $v_{q} \in J_{L}^{-1}(\mu)$ be a representative. The point $F\left(v_{[q]_{G}},[q]_{G_{\mu}}\right)$ is obtained by taking the consecutive quotients of $v_{q}$. In particular, the component of the final quotient in the configuration space $\left(Q / K_{\nu}\right) /\left(\bar{G}_{\nu}\right)$ of the magnetic Lagrangian system, is precisely the image $\tau$.

Since $F$ is a diffeomorphism, $\tau$ is onto. The Hamiltonian vector field on $T_{Q / G_{\mu}}(Q / G)$ and $T_{\left(Q / K_{\nu}\right) /\left(\bar{G}_{\nu}\right)_{\rho}}\left((Q / K) / \bar{G}_{\nu}\right)$ are $F$-related. Their integral curves project onto solutions of the Euler-Lagrange equations. This concludes the proof of Theorem 7 .

\section{$7 \quad$ Examples}

\subsection{Elroy's Beanie}

This system appears in e.g. [13]. It consists of two planar rigid bodies that are connected in their center of mass. The system moves in the plane and it is subject to some conservative force with potential $V$. The configuration space is $S E(2) \times S^{1}$, with coordinates $(x, y, \theta, \psi)$. Here $(x, y)$ is the position of the center of mass, $\theta$ is the rotation of the first rigid body, and $\psi$ the relative rotation of the second body w.r.t. the first. The kinetic energy of the system is $S E(2)$-invariant and we will suppose that the potential is invariant as well. This means in fact that only the relative position of the two bodies matters for the dynamics of the system. The Lagrangian is of the form

$$
L=\frac{1}{2} m\left(\dot{x}^{2}+\dot{y}^{2}\right)+\frac{1}{2} I_{1} \dot{\theta}^{2}+\frac{1}{2} I_{2}(\dot{\theta}+\dot{\psi})^{2}-V(\psi) .
$$

The Euler-Lagrange equations of the system are, in normal form,

$$
\ddot{x}=0, \quad \ddot{y}=0, \quad \ddot{\theta}=\frac{1}{I_{1}} V^{\prime}, \quad \ddot{\psi}=-\frac{I_{1}+I_{2}}{I_{1} I_{2}} V^{\prime} .
$$

The symmetry group and the principal connection. An element of $S E(2)$, the special Euclidean group, can be represented by a matrix of the form

$$
\left(\begin{array}{ccc}
\cos \theta & -\sin \theta & x \\
\sin \theta & \cos \theta & y \\
0 & 0 & 1
\end{array}\right)
$$

The identity of the group is $(x=0, y=0, \theta=0)$ and the multiplication is given by

$$
\left(x_{1}, y_{1}, \theta_{1}\right) *\left(x_{2}, y_{2}, \theta_{2}\right)=\left(x_{2} \cos \theta_{1}-y_{2} \sin \theta_{1}+x_{1}, x_{2} \sin \theta_{1}+y_{2} \cos \theta_{1}+y_{1}, \theta_{1}+\theta_{2}\right) \text {. }
$$

The matrices

$$
e_{1}=\left(\begin{array}{ccc}
0 & 0 & 1 \\
0 & 0 & 0 \\
0 & 0 & 0
\end{array}\right), \quad e_{2}=\left(\begin{array}{ccc}
0 & 0 & 0 \\
0 & 0 & 1 \\
0 & 0 & 0
\end{array}\right), \quad e_{3}=\left(\begin{array}{ccc}
0 & -1 & 0 \\
1 & 0 & 0 \\
0 & 0 & 0
\end{array}\right),
$$


form a basis for the Lie algebra, for which $\left[e_{1}, e_{2}\right]=0,\left[e_{1}, e_{3}\right]=e_{2}$ and $\left[e_{2}, e_{3}\right]=-e_{1}$. A corresponding basis of fundamental vector fields on $Q$ is

$$
\tilde{e}_{1}=\frac{\partial}{\partial x}, \quad \tilde{e}_{2}=\frac{\partial}{\partial y}, \quad \tilde{e}_{3}=-y \frac{\partial}{\partial x}+x \frac{\partial}{\partial y}+\frac{\partial}{\partial \theta},
$$

and a basis of invariant vector fields is

$$
\hat{e}_{1}=\cos \theta \frac{\partial}{\partial x}+\sin \theta \frac{\partial}{\partial y}, \quad \hat{e}_{2}=-\sin \theta \frac{\partial}{\partial x}+\cos \theta \frac{\partial}{\partial y}, \quad \hat{e}_{3}=\frac{\partial}{\partial \theta} .
$$

One can easily verify that the Lagrangian is invariant under the $S E(2)$-action. There is a trivial principal connection on $P=Q=S E(2) \times S^{1} \rightarrow Q / S E(2)=S^{1}$, which locally takes the form

$$
(d x+y d \theta) e_{1}+(d y-x d \theta) e_{2}+d \theta e_{3} .
$$

The momentum map $J_{L}$ is given by:

$$
J_{L}=m \dot{x} e^{1}+m \dot{y} e^{2}+\left(m(x \dot{y}-y \dot{x})+I_{1} \dot{\theta}+I_{2}(\dot{\theta}+\dot{\psi})\right) e^{3} .
$$

In what follows we perform two Routh reductions on the Lagrangian. The first reduction is done w.r.t. the full symmetry group $S E(2)$, and the second reduction w.r.t. the Abelian normal subgroup $\mathbb{R}^{2}$.

Full reduction. Let $\mu=\mu_{1} e^{1}+\mu_{2} e^{2}+\mu_{3} e^{3}$ be a generic element in $\mathfrak{g}^{*}$. An element $\xi=\xi^{1} e_{1}+\xi^{2} e_{2}+\xi^{3} e_{3}$ of the isotropy algebra $\mathfrak{g}_{\mu}$ satisfies

$$
\xi^{3} \mu_{2}=0, \quad \xi^{3} \mu_{1}=0, \quad \xi^{1} \mu_{2}-\xi^{2} \mu_{1}=0 .
$$

So if we suppose that $\mu_{1}$ and $\mu_{2}$ do not both vanish - we will set $\mu_{1}=1$ from now on - then a typical element of $\mathfrak{g}_{\mu}$ is $\xi=\xi^{1}\left(e_{1}+\mu_{2} e_{2}\right)$. Since $\mathfrak{g}_{\mu}$ is 1-dimensional, $G_{\mu}$ is of course Abelian. A convenient way to describe the manifold $P / G_{\mu}=S E(2) / G_{\mu}$ locally is by considering a coordinate transformation $\left(x^{\prime}, y^{\prime}, \theta^{\prime}\right)$ in the group coordinates such that the vector field associated to an element in $\mathfrak{g}_{\mu}$ becomes a coordinate vector field: in the new coordinates, we should have $\partial_{x^{\prime}}=\partial_{x}+\mu_{2} \partial_{y}$. This is obtained by the following transformation

$$
x^{\prime}=x, \quad y^{\prime}=y-\mu_{2} x, \quad \theta^{\prime}=\theta .
$$

Then clearly $\left(y^{\prime}, \theta\right)$ is a coordinate chart on $S E(2) / G_{\mu}$. And simultaneously, we have that $\left(y^{\prime}, \theta, \psi\right)$ is a coordinate chart on the reduced configuration manifold $P / G_{\mu}=\left(S E(2) \times S^{1}\right) / G_{\mu}$, and the fibration $P / G_{\mu} \rightarrow Q / G=S^{1}$ is locally represented by $\left(y^{\prime}, \theta, \psi\right) \mapsto(\psi)$. For a more systematic treatment on appropriate coordinate changes, we refer to [6].

We now compute the Routhian $L_{0}$ and the 2 -form $\mathcal{B}_{0}$. Following [19], a convenient way to compute the (unreduced) Routhian for mechanical Lagrangians is by using $2\left(L_{0}+V\right)=$ $\left(-p_{x} \dot{x}-p_{y} \dot{y}-\dot{\theta} p_{\theta}+\dot{\psi} p_{\psi}\right)_{J_{L}^{-1}(\mu)}$, where $p_{i}$ is the momentum in the $i$ th coordinate. We have:

$$
\begin{aligned}
2\left(L_{0}+V\right) & =\left(-m\left(\dot{x}^{2}+\dot{y}^{2}\right)-I_{1} \dot{\theta}^{2}-I_{2}(\dot{\theta}+\dot{\psi}) \dot{\theta}+I_{2}(\dot{\theta}+\dot{\psi}) \dot{\psi}\right)_{J_{L}^{-1}(\mu)} \\
& =\left(-m\left(\dot{x}^{2}+\dot{y}^{2}\right)-\left(I_{1}+I_{2}\right) \dot{\theta}^{2}+I_{2} \dot{\psi}^{2}\right)_{J_{L}^{-1}(\mu)} \\
& =-\frac{1}{m}\left(1+\mu_{2}^{2}\right)-\frac{\left(\mu_{3}-\left(x \mu_{2}-y\right)-I_{2} \dot{\psi}\right)^{2}}{I_{1}+I_{2}}+I_{2} \dot{\psi}^{2} \\
& =\frac{I_{1} I_{2}}{I_{1}+I_{2}} \dot{\psi}^{2}+2 I_{2} \frac{\mu_{3}-\left(x \mu_{2}-y\right)}{I_{1}+I_{2}} \dot{\psi}-\frac{\left(\mu_{3}-\left(x \mu_{2}-y\right)\right)^{2}}{I_{1}+I_{2}} .
\end{aligned}
$$


In the last step we have left out some constant terms. The reduced Lagrangian is then obtained by taking the quotient w.r.t. the action of $G_{\mu}$. This is done by applying the coordinate transformation introduced above. We get:

$$
L_{0}=\frac{1}{2} \frac{I_{1} I_{2}}{I_{1}+I_{2}} \dot{\psi}^{2}+I_{2} \frac{\mu_{3}+y^{\prime}}{I_{1}+I_{2}} \dot{\psi}-\left(V(\psi)+\frac{1}{2} \frac{\left(\mu_{3}+y^{\prime}\right)^{2}}{I_{1}+I_{2}}\right),
$$

which is clearly independent of $x^{\prime}$. The 2 -form $\mathcal{B}_{0}$ is obtained by reducing the 2 -form

$$
d\left((d x+y d \theta)+\mu_{2}(d y-x d \theta)+\mu_{3} d \theta\right)=d\left(y-\mu_{2} x\right) \wedge d \theta .
$$

Using the coordinate change we get $\mathcal{B}_{0}=d y^{\prime} \wedge d \theta$.

In this example the Routhian $L_{0}$ depends on the velocity corresponding to the coordinate $\psi$ on $S^{1}$, but is independent of the velocities corresponding to the two remaining coordinates $\left(y^{\prime}, \theta\right)$. With the above, the reduced Euler-Lagrange equations take the form

$$
\begin{aligned}
& \dot{y}^{\prime}=0, \\
& \dot{\theta}=\frac{1}{I_{1}+I_{2}}\left(y^{\prime}+\mu_{3}-I_{2} \dot{\psi}\right), \\
& \ddot{\psi}=-\frac{I_{1}+I_{2}}{I_{1} I_{2}} V^{\prime}-\frac{1}{I_{1}} \dot{y}^{\prime} .
\end{aligned}
$$

Note that the second order equation in $\psi$ decouples from the first order equations, and that these two first order equations are the momentum equations rewritten in normal form.

Abelian reduction. We now perform first Routh reduction w.r.t. the Abelian symmetry group $\mathbb{R}^{2}$ of translations in the $x$ and $y$ direction. Let us denote the symmetry group by $K=\mathbb{R}^{2}$ and study the quotient spaces. We will use the same notations as before: the Lie algebra elements $e_{1}, e_{2}$ denote a basis for the subalgebra $\mathfrak{K}$ of $K$ in $\mathfrak{g}$. The momentum map for this action is now $J_{K}=i^{*} \circ J_{L}=m \dot{x} e^{1}+m \dot{y} e^{2}$. We choose $\nu$ to be the projection of the momentum $\mu$ we had used in the full reduction: let $\nu=e^{1}+\mu_{2} e^{2} \in \mathfrak{g}^{*}$. Since $K$ is Abelian, $K_{\nu}=K$ and the quotient space is $S E(2) \times S^{1} / \mathbb{R}^{2}=S^{1} \times S^{1}$. If we choose $\mathcal{A}^{1}=d x e_{1}+d y e_{2}$ to be the trivial connection, we simply get $\mathcal{B}_{1}=0$. The Routhian $L_{1}$ can now be obtained from

$$
\begin{aligned}
2\left(L_{1}+V\right) & =\left(-p_{x} \dot{x}-p_{y} \dot{y}+p_{\theta} \dot{\theta}+p_{\psi} \dot{\psi}\right)_{J_{L}^{-1}(\nu)} \\
& =\left(-m \dot{x}^{2}-m \dot{y}^{2}+I_{1} \dot{\theta}^{2}+I_{2}(\dot{\theta}+\dot{\psi})^{2}\right)_{J_{L}^{-1}(\nu)}=I_{1} \dot{\theta}^{2}+I_{2}(\dot{\theta}+\dot{\psi})^{2},
\end{aligned}
$$

where we ignored again some constant terms. The Routh reduced system is now a standard Lagrangian system on $S^{1} \times S^{1}$ with Lagrangian $L_{1}=\frac{1}{2} I_{1} \dot{\theta}^{2}+\frac{1}{2} I_{2}(\dot{\theta}+\dot{\psi})^{2}-V(\psi)$ (see the paragraphs on Abelian Routh reduction). Its equations of motion are

$$
\ddot{\theta}=\frac{1}{I_{1}} V^{\prime}, \quad \ddot{\psi}=-\frac{I_{1}+I_{2}}{I_{1} I_{2}} V^{\prime} .
$$

For this example there is actually no second stage: the group $\bar{G}_{\nu}=G_{\nu} / K_{\nu}$ is the trivial one $\{e\}$, and the vector space $\overline{\mathfrak{g}}_{\nu}=\mathfrak{g}_{\nu} / \mathfrak{K}_{\nu}$ is only the zero vector. So, there is no second momentum map to take into account, and there is no further symmetry to quotient out.

In the reduction by stages process we have not made use of $\mu_{3}$. We now show that the two ways of reducing the system are equivalent.

Equivalence between direct reduction and reduction by stages. Let us compute the diffeomorphism $F$ for this example. Here, it is a map $J_{L}^{-1}(\mu) / G_{\mu} \rightarrow J_{K}^{-1}(\nu) / K$ that is obtained by projection of the inclusion map $J_{L}^{-1}(\mu) \rightarrow J_{K}^{-1}(\nu)$. The latter equals, in coordinates

$$
(x, y, \theta, \psi, \dot{\psi}) \mapsto\left(x, y, \theta, \psi, \dot{\theta}=\frac{1}{I_{1}+I_{2}}\left(\mu_{3}-\left(x \mu_{2}-y\right)-I_{2} \dot{\psi}\right), \dot{\psi}\right) .
$$


The map is reducible, and after taking the quotient it becomes

$$
F: J_{L}^{-1}(\mu) / G_{\mu} \rightarrow J_{K}^{-1}(\nu) / K ; \quad\left(y^{\prime}, \theta, \psi, \dot{\psi}\right) \mapsto\left(\theta, \psi, \dot{\theta}=\frac{1}{I_{1}+I_{2}}\left(\mu_{3}+y^{\prime}-I_{2} \dot{\psi}\right), \dot{\psi}\right) .
$$

This diffeomorphism maps the $G_{\mu}$-reduced system on the $K_{\nu}$-reduced system, as is obvious from the respective equations of motion.

\subsection{Rigid bodies on the Heisenberg group}

As a second example of Routh reduction by stages, we discuss the dynamics of a rigid body immersed in a potential flow with circulation [20]. We assume that the body is circular, and in this case the equations of motion are given by

$$
\frac{d}{d t}\left[\begin{array}{c}
p_{x} \\
p_{y}
\end{array}\right]=\Gamma\left[\begin{array}{c}
-v_{y} \\
v_{x}
\end{array}\right], \quad \text { where } \quad\left[\begin{array}{l}
p_{x} \\
p_{y}
\end{array}\right]=\mathbb{M}\left[\begin{array}{l}
v_{x} \\
v_{y}
\end{array}\right]
$$

Here $\Gamma$ represents the circulation and $\mathbb{M}$ is a (non-diagonal) mass matrix, which incorporates the inertia and added masses of the body. The right-hand side of the equations of motion represents the so-called Kutta-Joukowski lift force, a gyroscopic force due to circulation [9, 17].

While this system is extremely easy to integrate, it nevertheless exhibits all the interesting geometric characteristics of more complicated examples. As we show below, the configuration space for this system is the Heisenberg group, arguably the simplest non-trivial central extension group, and the procedure of reduction by stages demonstrated here can be applied equally well to more complicated central extensions, such as the oscillator group (describing the dynamics of rigid bodies of arbitrary cross section in circulatory flow) and the Bott-Virasoro group describing the KdV equation.

In this context, the Heisenberg group $H$ is the Euclidian space $\mathbb{R}^{3}$, equipped with the multiplication

$$
(x, y, s) \cdot\left(x^{\prime}, y^{\prime}, s^{\prime}\right)=\left(x+x^{\prime}, y+y^{\prime}, s+s^{\prime}+\frac{1}{2}\left(x y^{\prime}-y x^{\prime}\right)\right),
$$

using the definition of $H$ given in [18]. The coordinates $(x, y)$ describe the center of the circular disc, while the coordinate $s$ corresponds to the flux of the fluid around the body. The conjugate momentum corresponding to $s$ will be the circulation of the fluid.

The Heisenberg group can alternatively be described as the central extension of $\mathbb{R}^{2}$ constructed by means of the cocycle $B\left((x, y),\left(x^{\prime}, y^{\prime}\right)\right)=\frac{1}{2}\left(x y^{\prime}-y x^{\prime}\right)$. We now introduce the infinitesimal cocycle $C: \mathbb{R}^{2} \times \mathbb{R}^{2} \rightarrow \mathbb{R}$, given by

$$
C\left(\left(v_{x}, v_{y}\right),\left(v_{x}^{\prime}, v_{y}^{\prime}\right)\right)=v_{x} v_{y}^{\prime}-v_{y} v_{x}^{\prime} .
$$

For the relation between $B$ and $C$, see for instance [12]. The Lie algebra $\mathfrak{h}$ of $H$ can then be identified with $\mathbb{R}^{3}$ with the bracket

$$
\left[\left(v_{x}, v_{y}, v\right),\left(v_{x}^{\prime}, v_{y}^{\prime}, v^{\prime}\right)\right]=\left(0,0, C\left(\left(v_{x}, v_{y}\right),\left(v_{x}^{\prime}, v_{y}^{\prime}\right)\right)\right)=\left(0,0, v_{x} v_{y}^{\prime}-v_{y} v_{x}^{\prime}\right) .
$$

The dual Lie algebra $\mathfrak{h}^{*}$ can again be identified with $\mathbb{R}^{3}$, with coordinates $\left(p_{x}, p_{y}, p\right)$ and duality pairing $\left\langle\left(p_{x}, p_{y}, p\right),\left(v_{x}, v_{y}, v\right)\right\rangle=p_{x} v_{x}+p_{y} v_{y}+p v$.

We now induce the following quadratic Lagrangian $\ell$ on $\mathfrak{h}$ :

$$
\ell\left(v_{x}, v_{y}, v\right)=\frac{1}{2}\left[v_{x}, v_{y}\right] \mathbb{M}\left[\begin{array}{l}
v_{x} \\
v_{y}
\end{array}\right]+\frac{v^{2}}{2},
$$


and we define $L$ on $H$ by left extension: $L(g, \dot{g})=\ell\left(g^{-1} \dot{g}\right)$, or explicitly

$$
L(x, y, s ; \dot{x}, \dot{y}, \dot{s})=\frac{1}{2}[\dot{x}, \dot{y}] \mathbb{M}\left[\begin{array}{c}
\dot{x} \\
\dot{y}
\end{array}\right]+\frac{1}{2}\left(\dot{s}-\frac{1}{2}(x \dot{y}-y \dot{x})\right)^{2} .
$$

The Euler-Poincaré equations obtained from $\ell$ are given by

$$
\frac{d}{d t}\left[\begin{array}{c}
p_{x} \\
p_{y} \\
p
\end{array}\right]=p\left[\begin{array}{c}
-v_{y} \\
v_{x} \\
0
\end{array}\right]
$$

After setting $p=\Gamma$, we obtain the equations (7). These equations also coincide with the EulerLagrange equations obtained from the Lagrangian (8).

Reduction with respect to $\mathbb{R}$. The center of $H$ is the normal subgroup isomorphic to $\mathbb{R}$ which consists of all elements of the form $(0,0, s)$, where $s \in \mathbb{R}$. We first perform Routh reduction with respect to the left action of this subgroup on $H$. On the principal bundle $H \rightarrow H / \mathbb{R} \cong \mathbb{R}^{2}$ we consider the connection one-form given at the identity by $\mathcal{A}(e)\left(v_{x}, v_{y}, v\right)=v$, and extended to the whole of $H$ by left translation. Explicitly, we have

$$
\mathcal{A}(x, y, s)=d s-\frac{1}{2}(x d y-y d x) .
$$

Since the structure group $\mathbb{R}$ is Abelian, the curvature of $\mathcal{A}$ is given by $\mathcal{B}=d \mathcal{A}=-d x \wedge d y$.

Similarly, the momentum map $J_{L}: T H \rightarrow \mathbb{R}$ for the $\mathbb{R}$-action on the tangent bundle $T H$ is given by $J_{L}(x, y, z ; \dot{x}, \dot{y}, \dot{z})=\dot{s}-(x \dot{y}-y \dot{x}) / 2$, so that $J_{L}^{-1}$ consists of all points $(x, y, s ; \dot{x}, \dot{y}, \dot{s})$ with $\dot{s}=\Gamma+(x \dot{y}-y \dot{x}) / 2$. The isotropy subgroup $\mathbb{R}_{\Gamma}=\mathbb{R}$ acts on this level set by translations in the $s$-direction, so that the reduced velocity space is given by

$$
J^{-1}(\Gamma) / \mathbb{R}_{\Gamma}=T \mathbb{R}^{2} .
$$

The symplectic form on the reduced space can easily be computed, and is explicitly given by

$$
A d \dot{x} \wedge d x+B(d \dot{x} \wedge d y+d \dot{y} \wedge d x)+C d \dot{y} \wedge d y-\Gamma d x \wedge d y
$$

where $A, B, C$ are the entries of the mass matrix $\mathbb{M}$. The last term of the symplectic form, $-\Gamma d x \wedge d y$, is the curvature term of the connection, paired with $\Gamma \in \mathbb{R}$. Finally, a quick computation shows that the reduced Lagrangian is just the kinetic energy Lagrangian on $T \mathbb{R}^{2}$ :

$$
L_{1}(x, y ; \dot{x}, \dot{y})=\frac{1}{2}[\dot{x}, \dot{y}] \mathbb{M}\left[\begin{array}{c}
\dot{x} \\
\dot{y}
\end{array}\right]
$$

up to constant terms.

Second reduction. We now perform reduction with respect to the remaining symmetry group, $H / \mathbb{R} \cong \mathbb{R}^{2}$, using the results from Section 5.3. We have a left invariant magnetic Lagrangian system on the group $\mathbb{R}^{2}$, with Lagrangian (9) and magnetic form $\mathcal{B}_{\Gamma}=-\Gamma d x \wedge d y$. The potential $\delta: \mathbb{R}^{2} \rightarrow \mathbb{R}^{2}$ corresponding to the latter is given by

$$
\delta(x, y)=\Gamma\left[\begin{array}{c}
-y \\
x
\end{array}\right]
$$

and the momentum map is therefore $J_{2}(x, y, \dot{x}, \dot{y})=\mathbb{M}(\dot{x}, \dot{y})^{T}-\delta(x, y)$. The non-equivariance 2-cocycle of the momentum map is $\Sigma_{\delta}=\mathcal{B}_{\Gamma}$.

The affine action of $\mathbb{R}^{2}$ on itself is given by $(x, y) \cdot\left(p_{x}, p_{y}\right)=\left(p_{x}-\Gamma y, p_{y}+\Gamma x\right)$. If we fix a momentum value $(\lambda, \mu) \in \mathbb{R}^{2}$, the isotropy group $\mathbb{R}_{(\lambda, \mu)}^{2}$ of the affine action consists of just the zero element, and consequentially the twice-reduced space $J_{2}^{-1}(\lambda, \mu) / \mathbb{R}_{(\lambda, \mu)}^{2}$ is nothing but $\mathbb{R}^{2}$. 
The reduced Euler-Lagrange equations (3) in the case of a left action, assume the following form

$$
\frac{d}{d t}\left[\begin{array}{l}
p_{x} \\
p_{y}
\end{array}\right]=-i_{\left(v_{x}, v_{y}\right)} \Sigma_{\delta}=\Gamma\left[\begin{array}{c}
-v_{y} \\
v_{x}
\end{array}\right]
$$

with $\left(p_{x}, p_{y}\right)^{T}=\mathbb{M}\left(v_{x}, v_{y}\right)^{T}$, and these are nothing but the equations (7).

\section{Acknowledgements}

BL is an honorary postdoctoral researcher at the Department of Mathematics of Ghent University and associate academic staff at the Department of Mathematics of K.U.Leuven. BL is sponsored by a Research Programme of the Research Foundation - Flanders (FWO). Part of this work was supported by the Sint-Lucas department of Architecture, K.U.Leuven Association. TM is a Postdoctoral Fellow of the Research Foundation - Flanders (FWO). JV is a postdoc at the Department of Mathematics of UC San Diego, partially supported by NSF CAREER award DMS-1010687 and NSF FRG grant DMS-1065972, and is on leave from a Postdoctoral Fellowship of the Research Foundation-Flanders. This work is part of the IRSES project GEOMECH (nr. 246981) within the 7th European Community Framework Programme. We are indebted to F. Cantrijn, M. Crampin and E. García-Toraño Andres for many useful discussions. We thank one of the referees for pointing out reference [16] on the reduction hypothesis.

\section{References}

[1] Abraham R., Marsden J.E., Foundations of mechanics, Benjamin/Cummings Publishing Co., Inc., Advanced Book Program, Reading, Mass., 1978.

[2] Adamec L., A route to Routh - the classical setting, J. Nonlinear Math. Phys. 18 (2011), 87-107.

[3] Arnold V.I., Kozlov V.V., Neishtadt A.I., Mathematical aspects of classical and celestial mechanics, SpringerVerlag, Berlin, 1997.

[4] Cendra H., Marsden J.E., Ratiu T.S., Lagrangian reduction by stages, Mem. Amer. Math. Soc. 152 (2001), no. 722 .

[5] Cortés J., de León M., Marrero J.C., Martín de Diego D., Martínez E., A survey of Lagrangian mechanics and control on Lie algebroids and groupoids, Int. J. Geom. Methods Mod. Phys. 3 (2006), 509-558, math-ph/0511009.

[6] Crampin M., Mestdag T., Routh's procedure for non-Abelian symmetry groups, J. Math. Phys. 49 (2008), 032901, 28 pages, arXiv:0802.0528.

[7] Echeverría-Enríquez A., Muñoz-Lecanda M.C., Román-Roy N., Reduction of presymplectic manifolds with symmetry, Rev. Math. Phys. 11 (1999), 1209-1247, math-ph/9911008.

[8] Kobayashi S., Nomizu K., Foundations of differential geometry, Vol. I, Interscience Publishers, New York London, 1963.

Kobayashi S., Nomizu K., Foundations of differential geometry, Vol. II, Interscience Publishers, New York London, 1969.

[9] Lamb H., Hydrodynamics, Reprint of the 1932 6th ed., Cambridge Mathematical Library, Cambridge University Press, Cambridge, 1993.

[10] Langerock B., Cantrijn F., Vankerschaver J., Routhian reduction for quasi-invariant Lagrangians, J. Math. Phys. 51 (2010), 022902, 20 pages, arXiv:0912.0863.

[11] Langerock B., Castrillón Lopéz M., Routh reduction for singular Lagrangians, Int. J. Geom. Methods Mod. Phys. 7 (2010), 1451-1489, arXiv:1007.0325.

[12] Marsden J.E., Misiołek G., Ortega J.P., Perlmutter M., Ratiu T.S., Hamiltonian reduction by stages, Lecture Notes in Mathematics, Vol. 1913, Springer, Berlin, 2007.

[13] Marsden J.E., Montgomery R., Ratiu T.S., Reduction, symmetry, and phases in mechanics, Mem. Amer. Math. Soc. 88 (1990), no. 436. 
[14] Marsden J.E., Ratiu T.S., Scheurle J., Reduction theory and the Lagrange-Routh equations, J. Math. Phys. 41 (2000), 3379-3429.

[15] Mestdag T., Crampin M., Invariant Lagrangians, mechanical connections and the Lagrange-Poincaré equations, J. Phys. A: Math. Theor. 41 (2008), 344015, 20 pages, arXiv:0802.0146.

[16] Mikityuk I.V., Stepin A.M., A sufficient condition for stepwise reduction: proof and applications, Sb. Math. 199 (2008), 663-671.

[17] Milne-Thomson L., Theoretical hydrodynamics, 5th ed., MacMillan, London, 1968.

[18] Montgomery R., A tour of subriemannian geometries, their geodesics and applications, Mathematical Surveys and Monographs, Vol. 91, American Mathematical Society, Providence, RI, 2002.

[19] Pars L.A., A treatise on analytical dynamics, Heinemann Educational Books Ltd., London, 1965.

[20] Vankerschaver J., Kanso E., Marsden J.E., The dynamics of a rigid body in potential flow with circulation, Regul. Chaotic Dyn. 15 (2010), 606-629, arXiv:1003.0080. 